\title{
An LES-PBE-PDF approach for predicting the soot particle size distribution in turbulent flames
}

\author{
Fabian Sewerin and Stelios Rigopoulos*
}

Department of Mechanical Engineering, Imperial College London, Exhibition Road, London SW7 2AZ, UK

\begin{abstract}
In this article, we combine the large eddy simulation (LES) concept with the population balance equation (PBE) for predicting, in a Eulerian fashion, the evolution of the soot particle size distribution in a turbulent non-premixed hydrocarbon flame. In order to resolve the interaction between turbulence and chemical reactions/soot formation, the transport equations for the gas phase scalars and the PBE are combined into a joint evolution equation for the filtered $p d f$ associated with a single realization of the gas phase composition and the soot number density distribution. With view towards an efficient numerical solution procedure, we formulate Eulerian stochastic field equations that are statistically equivalent to the joint scalar-number density $p d f$. By discretizing the stochastic field equation for the particle number density using an explicit adaptive grid technique, we are able to accurately resolve sharp features of evolving particle size distributions, while keeping the number of grid points in particle size space small.

Compared to existing models, the main advantage of our approach is that the LES-filtered particle size distribution is predicted at each location in the flow domain and every instant in time and that arbitrary chemical reaction mechanisms and soot formation kinetics can be accommodated without approximation.

The combined LES-PBE-PDF model is applied to investigate soot formation in the turbulent non-premixed Delft III flame. Here, the soot kinetics encompass acetylene-based rate expressions for nucleation and growth that were previously employed in the context of laminar diffusion flames. In addition, both species consumption by soot formation and radiation based on the assumption of optical thinness are accounted for. While the agreement of our model predictions with experimental measurements is not perfect, we indicate the benefits of the LES-PBE-PDF model and demonstrate its computational viability.
\end{abstract}

Keywords: LES, Population balance, PDF methods, Soot formation

\section{Introduction}

Soot particles form in hydrocarbon combustion devices and are typically created in fuel-rich regions of high temperature. From an engineering perspective, soot particles contribute a heat sink through thermal radiation, but may also pollute a combustor if deposited on its walls. On the other hand, soot particles are known to be harmful to the environment, acting as greenhouse agents, and to the human body as they may cause respiratory diseases [28] or act as carcinogens. By consequence, many of the recent modelling efforts in the combustion community have targeted the prediction of soot formation in hydrocarbon combustion, taking into account the effect of soot on the flame structure.

Typically, soot is considered as a particulate phase that is polydispersed with respect to particle size and behaves non-inertially, that is, the individual soot particles are assumed to be small enough such that they respond instantaneously to changes in the carrier flow field. From a Eulerian perspective, the soot phase can be described by the population balance equation (PBE) which governs the evolution of the soot particle size distribution throughout the flow domain and is formulated in terms of the number density of soot particles per unit volume of mixture and unit of length in particle size space.

In this article, we present a comprehensive LES-PBE-PDF approach for predicting the evolution of the soot particle size distribution in a turbulent flame. The main ingredient of our model is an evolution equation for the LES-filtered one-point, one-time joint probability density function $(p d f)$ associated with a single realization of the reactive gas phase scalars and the particle number density. Here, the physical processes related to chemical reactions, soot particle inception, soot growth and coagulation appear in closed form, while velocity and two-point correlations require modelling. For these, we adopt a standard gradient diffusion hypothesis as well as an IEM-based (interaction by exchange with the mean) micromixing model.

\footnotetext{
*Corresponding author (s.rigopoulos@imperial.ac.uk)
} 
Originally, the PBE-PDF concept was introduced by Rigopoulos [65] who showed that, upon discretization in particle size space, the PBE reduces to a collection of particle phase scalars which may be incorporated into a transported $p d f$ approach in the same way as the reactive scalars describing the gas phase [66]. This leads to a transport equation for the so-called joint scalar-discrete number density $p d f$ which naturally accounts for all one-point, one-time correlations of the gas phase composition and the discrete number densities. In the context of RANS, Di Veroli and Rigopoulos [11, 12] showed that a numerical solution of this transport equation based on a stochastic particle method is computationally feasible for practical flow configurations and realistic nucleation/growth kinetics, albeit expensive. Subsequently, Akridis and Rigopoulos [3] and Akridis [2] applied the RANS-PBE-PDF methodology to investigate soot formation in two turbulent diffusion flames.

More recently, the PBE-PDF concept has also been combined with LES and, coincidentally, different strategies have emerged for reducing the computational expense associated with conventional solution schemes of the $p d f$ transport equation. Neuber et al. [54], for instance, devised a sparse stochastic particle solution scheme by invoking the generalized multiple mapping conditioning (MMC) method. From a physical perspective, the MMC-LES approach is based on shifting micromixing locality from physical space to the space spanned by the reactive scalars and discrete number densities. Sewerin and Rigopoulos [69], on the other hand, revisited the PBE-PDF rationale and formulated a joint scalar-number density $p d f$ transport equation that is independent of a specific particle size discretization. An important consequence is that particle size persists as an independent coordinate in a statistically equivalent stochastic particle or field reformulation and that these stochastic systems may hence be discretized with respect to particle size using both fixed and adaptive grid schemes.

In the present article, we generalize this approach to polydispersed particle formation in variable density flows of gases at low Mach numbers. The joint scalar-number density $p d f$ transport equation which we obtain is solved numerically by the method of Eulerian stochastic fields [26, 67, 72] and, for the discretization in particle size space of the stochastic field equation associated with the particle size distribution, an explicit adaptive grid technique is applied [68].

By construction, the PBE-PDF approach achieves a direct resolution of the particle size distribution. This is different from moment-based approaches [61] in which the particle size distribution is described in terms of a small number of low order statistical moments such as the particle number or volume density. Moment-based methods are well-established by now and are not only computationally very economical, but also readily generalize to situations in which the particulate phase is characterized by more than one characteristic property. However, the main challenge associated with these formulations is that the moment equations are closed only for particular functional forms of the particle growth rate and the coagulation kernel. Common closure schemes such as the quadrature method of moments involve an assumption on the shape of the particle size distribution which allows for truncated moments to be expressed in terms of the first few resolved moments.

The scientific contribution of our work is threefold: First, we develop an LES-based model for predicting the evolution of the soot particle size distribution in a turbulent combusting flow at low Mach number. An integral part of our approach is the PBE-PDF closure of the interaction between turbulence and chemical reactions/particle formation which allows for the incorporation of arbitrary gas phase and soot kinetics without approximation. Second, we present a stochastic field formulation that reproduces, in a statistical sense, the evolution of the joint scalar-number density $p d f$ and can be combined with both fixed and adaptive grid discretization schemes along the particle size coordinate. Finally, the computational viability and predictive capabilities of the combined LES-PBE-PDF approach are demonstrated in the context of the Delft III turbulent diffusion flame. In particular, we show that a detailed resolution of the soot particle size distribution hardly increases the computational cost and that the overall model is computationally feasible on modern computing devices. Furthermore, to our awareness, the present modelling effort constitutes the first attempt to directly predict soot particle size distributions within the scope of LES.

This article is organized as follows: In Section 2, we first review existing modelling strategies for predicting soot formation in turbulent non-premixed flames. Subsequently, in Section 3, the PBE and the LES concept are formally introduced and an evolution equation for the joint scalar-number density $p d f$ is obtained. Here, we also discuss the micromixing closure and formulate the stochastic field equations. This is followed by Section 4, where the gas phase and soot kinetics and the radiation model are detailed. In Sections 5 and 6, we summarize details on the Delft III flame as well as the computer implementation used in this work. Model predictions are compared with experimental measurements from the Delft III database in Section 7 and discussed in the light of previous modelling attempts. Finally, we offer 
conclusions in Section 8 and provide a view towards further model enhancements.

\section{Approaches for modelling soot formation in turbulent non- premixed flames}

In the present section, we briefly review existing approaches for modelling soot formation in turbulent non-premixed flames. As an aid to the reader, the references discussed here are classified in Table 1.

From a modelling perspective, incorporating the formation of soot into models for turbulent reacting flows has been challenging for several main reasons. On the one hand, soot particles contribute significantly to radiative heat emission and, potentially, reabsorption, thus influencing the distribution of temperature and density in the carrier gas. On the other hand, the synthesis of soot precursors, the inception of soot and soot surface growth (both by PAH condensation and surface reactions) are characterized by much longer time scales than the mixing of reactants [5, 8, 74]. Attili et al. [5] argued that, as a consequence, the soot formation kinetics react only slowly to changes in the local turbulent mixing (scalar dissipation) rate. Furthermore, soot particles are characterized by a very low mass diffusivity and, contrary to light gas phase species, are mainly convected along by the ambient velocity field without significant dispersion [8].

Since RANS-based conserved scalar/presumed $p d f$ approaches are computationally very economical, an early idea for accommodating the first challenge mentioned above was to introduce an indicator for radiative heat losses into such a model. Following Gore et al. [20], Young and Moss [74] augmented a steady-state flamelet representation of the gas composition by a heat loss parameter such that the reactive scalars could formally be parameterized by a mixture fraction, the scalar dissipation rate and a heat loss coefficient. As a criterion for determining the heat loss coefficient, Young and Moss [74] proposed the condition that, locally, the Favre-averaged enthalpy computed from the extended flamelet library and the presumed mixture fraction $p d f$ coincides with the value obtained by solving a transport equation for the Favre-averaged mixture enthalpy with radiative heat losses. For the description of the soot particulate phase, Young and Moss [74] adopted the semi-empirical model of Moss et al. [46] based on two evolution equations for the Favre-averaged soot number density and volume fraction.

Conceptually, this approach is based on the premise that the carbon content of the gas phase (represented by the conserved mixture fraction) and the carbon represented by the soot-related scalars evolve independently. In heavily sooting flames, however, this may lead to an overprediction of the carbon content near soot pockets and, thus, to increased soot nucleation/growth rates. Furthermore, researchers have pointed out that the slow reaction rates associated with soot precursors entail a delayed response to changes in the scalar dissipation rate such that concentrations of soot precursors cannot be uniquely parameterized by mixture fraction and scalar dissipation rate as in a steady-state flamelet [48, 74]. Despite these concerns, the extended flamelet approach has been very influential in the past decades and motivated both model enhancement as well as application to several turbulent non-premixed flames. Bressloff et al. [9] incorporated the discrete transfer radiation model into the approach of Young and Moss [74] and assessed predictions of soot volume fraction in a confined turbulent methane-air diffusion flame. Bai et al. [7], on the other hand, adopted a one-equation semi-empirical soot model along with the hypothesis of optical thinness in order to investigate soot formation in a turbulent ethylene diffusion flame. Further to previous efforts, these authors considered the joint $p d f$ of mixture fraction and scalar dissipation rate and proposed a presumed $p d f$ model based on the product of a $\beta$-pdf for mixture fraction and a lognormal $p d f$ for the scalar dissipation rate. The extended flamelet approach combined with a presumed $\beta$ - $p d f$ method for mixture fraction was also adopted by Reddy et al. [64] who included non-gray radiation effects and considered the semi-empirical model by Brookes and Moss [10] for describing the evolution of the instantaneous soot number density and volume fraction. In order to obtain Favre-averages of these two quantities, Reddy et al. [64] computed expectations with respect to an additional presumed $p d f$ for temperature.

Formally, the evolution of the soot particle size (or, more generally, soot particle property) distribution through a flame can be described by the PBE which accounts for the physical processes by which soot particles interact both with the ambient gas phase (nucleation, surface growth, oxidation) and among each other (coagulation, aggregation). The semi-empirical one- and two-equation models referred to above are based on a moment reduction of the PBE for a monodisperse particle size distribution and include kinetic rate parameters that were estimated based on measurements in laminar flames [10, 74]. Conceptually, moment transformations of the PBE remain valid independent of the assumption of monodispersity, and 
for some functional forms of the soot growth/oxidation and coagulation rates, the moment equations are naturally closed at any order. For general kinetics, several approximate closure schemes have been developed [41, 61] and tailored to the characteristics of soot formation [47]. Physically, these closures represent an assumption on the shape of the particle size distribution, but maintain the kinetic details of the PBE and generalize to any number of moments.

Zucca et al. [75] applied the direct quadrature method of moments (DQMOM) and combined chemical equilibrium kinetics with a presumed $\beta$-pdf method for mixture fraction. Based on a moment reformulation of the bivariate PBE, Mueller and Pitsch [48] recently presented an LES-presumed pdf model which resolves the limitations of the original extended flamelet/presumed $p d f$ approach, while maintaining its computational efficiency. Specifically, these authors augmented the evolution equations for the gas phase scalars by source terms for radiative heat losses and species/element absorption and accommodated the slow chemistry of soot precursors by adding a separate transport equation for a 'lumped' PAH mass fraction.

Considering the same representation of the gas composition and soot as Mueller and Pitsch [48], Donde et al. [14] explored a transported $p d f$ approach based on the IEM micromixing model to resolve the turbulence-chemistry/particle formation interaction as compared to a presumed $p d f$ closure. In a subsequent investigation, Xuan and Blanquart [73] replaced the lumped PAH evolution equation in the original LES-presumed $p d f$ model of Mueller and Pitsch [48] by evolution equations for benzene and naphtalene and proposed a relaxation model to close the associated source terms under the LES viewpoint.

In transported $p d f$ methods, the scalar source terms appear naturally closed, while turbulent transport and molecular mixing of the gas phase and soot scalars require phenomenological closures [29]. In the context of soot formation in turbulent flames, transported $p d f$ methods seem to have first been introduced by Metternich et al. [45] who formulated a joint scalar-soot volume fraction $p d f$ transport equation based on a constrained equilibrium model of the thermochemistry and a semi-empirical model for the evolution of soot volume fraction. Subsequently, this approach was taken further by Lindstedt and Louloudi [39] who developed a kinetically detailed model for soot formation based on a moment-reformulation of the PBE and solved a transport equation for the joint scalar-moment $p d f$.

Intermediate in computational expense between presumed and transported $p d f$ methods for the turbulence-chemistry/soot formation interaction are multi-environment presumed $p d f$ methods $[15,16]$. Here, the joint scalar $p d f$ is represented by a linear combination of the instantaneous pdfs associated with several model flow realizations which exchange likelihood/volume fraction according to a particular micromixing prescription. In the so-called DQMOM-IEM approach, this micromixing model is determined such that the evolution and interaction of the model flow representations preserve the first unmixed moments of the joint scalar pdf. In the context of RANS, Reddy and De [63] combined the DQMOM-IEM approach for modelling the interaction of turbulence and gas phase chemistry with the semi-empirical soot model of Brookes and Moss [10] and analyzed the influence of several radiation models on predictions of soot volume fraction in two turbulent diffusion flames. Following their previous investigations [62, 64], these authors introduced a separate presumed $p d f$ for temperature and evaluated expectations of the temperature-dependent soot source terms with respect to this $p d f$.

In turbulent flames which do not experience extinction or reignition, the conditional moment closure approach $(\mathrm{CMC})$ can be a computationally economical alternative to transported $p d f$ methods. In the context of soot formation, Kronenburg et al. [37] seemed to have been the first to incorporate a semiempirical soot model into a RANS-CMC approach. Commensurate with the third challenge mentioned above, these authors demonstrated the importance of accounting for differential diffusion between the gas- and soot-describing scalars. Combining the same soot model with an LES-CMC approach, NavarroMartinez and Rigopoulos [52] similarly concluded that differential diffusion between the gas phase scalars and soot leads to more intermittent and locally intense soot volume fraction predictions.

Contrary to moment-based reductions of the PBE, only few researchers have attempted to resolve the soot particle size distribution in turbulent flames. An impending challenge, in this regard, is that mature primary soot particles tend to assemble in the form of chain-like aggregates. In order to describe these, the particle size coordinate needs to be complemented by an additional particle property such as a fractal dimension or the number of primary particles per aggregate. While a univariate description in terms of particle size is well-suited for tracing the evolution of primary soot particles or of aggregates with a fixed fractal shape, it is limited to shape-preserving particle formation and interaction processes.

In an a posteriori approach, soot particle size distributions are sometimes estimated by solving the PBE along specific paths through the flame, taking into account mean field information from a calculation 


\begin{tabular}{|c|c|c|c|c|c|}
\hline \multirow{2}{*}{$\begin{array}{l}\text { Representation of } \\
\text { the number } \\
\text { density } \\
\text { distribution }\end{array}$} & \multicolumn{5}{|c|}{ Turbulence-chemistry/particle formation interaction } \\
\hline & $\begin{array}{c}\text { Perfect } \\
\text { micromixing }\end{array}$ & $\begin{array}{c}\beta-/ \delta-/ \log - \\
\text { normal- } p d f\end{array}$ & $\begin{array}{l}\text { DQMOM- } \\
\text { IEM }\end{array}$ & $\mathrm{CMC}$ & $\begin{array}{c}\text { Transported } \\
p d f\end{array}$ \\
\hline $\begin{array}{l}\text { Semi-empirical } \\
\text { XMOMY } \\
\text { DPB } \\
\text { (Adaptive) FVM }\end{array}$ & {$[21,53]$} & $\begin{array}{l}{[7,9,62,64,74]} \\
{[75][35,48,73]^{\star}}\end{array}$ & {$[63]$} & {$[37][52]^{\star}$} & $\begin{array}{c}{[45]} \\
{[39][14]^{\star}} \\
{[2,3] \text { This work }}\end{array}$ \\
\hline
\end{tabular}

Table 1: Overview of different approaches for modelling soot formation in turbulent non-premixed flames (DPB: Discretized Population Balance, FVM: Finite Volume Method, XMOMY: Quadrature based Method of Moments, e.g., MOMIC, DQMOM, HMOM). The references marked by a superscript * indicate LES-based modelling approaches, while unmarked references adopted the RANS turbulence model. (In the classification, we associate approaches in which the soot size distribution was computed in a post-processing step based on mean field information with the 'perfect micromixing' assumption.)

without soot formation or with a moment-based soot model. Grosschmidt et al. [21], for instance, computed solutions to the PBE along streamlines of a reacting flow field obtained from a flamelet/presumed $p d f$ model of the gas phase combustion. A slightly different approach was followed by Netzell et al. [53] who reformulated the PBE in mixture fraction space and solved a series of unsteady flamelet problems for scalar dissipation rates sampled from the flame field predictions of Bai et al. [7].

The PBE-PDF concept which we adopt here achieves a direct resolution of the particle size distribution within the scope of a transported $p d f$ closure for the turbulence-chemistry and particle formation interaction [65]. In the context of RANS, this approach was recently applied to soot formation by Akridis and Rigopoulos [3] and Akridis [2] who investigated turbulent, non-premixed flames at atmospheric and elevated pressure. In the present article, the PBE-PDF paradigm is incorporated into an LES framework for combusting variable density flows at low Mach number. Our developments are guided by the objective to devise an efficient numerical solution scheme based on the stochastic field method and an explicit adaptive grid discretization in particle size space [68].

\section{Physical model}

\subsection{Governing equations}

In this section, we briefly review the conservation laws that are relevant to the continuum mechanical description of a fluid flow with an immersed particulate phase. In view of a Eulerian formulation, we introduce the instantaneous velocity $\mathbf{u}(\mathbf{x}, t)$, the pressure $p(\mathbf{x}, t)$ and the fluid density $\rho(\mathbf{x}, t)$ at a location $\mathbf{x}$ in a flow domain $\Omega$ and a point in time $t \geq t_{0}$. In Cartesian coordinates, the continuity and momentum balance laws can be written in the following form

$$
\begin{gathered}
\frac{\partial \rho}{\partial t}+\sum_{j=1}^{3} \frac{\partial \rho u_{j}}{\partial x_{j}}=0 \\
\frac{\partial \rho u_{i}}{\partial t}+\sum_{j=1}^{3} \frac{\partial \rho u_{i} u_{j}}{\partial x_{j}}=-\frac{\partial p}{\partial x_{i}}+\sum_{j=1}^{3} \frac{\partial \tau_{i j}}{\partial x_{j}}+\rho g_{i}, \quad i=1, \ldots, 3,
\end{gathered}
$$

where $\tau_{i j}$ denotes the viscous stress tensor of a Newtonian fluid and $\mathbf{g}$ is the gravitational acceleration.

Commonly, the carrier fluid is described in terms of reactive scalars $\mathbf{Y}(\mathbf{x}, t)$ which evolve according to

$$
\frac{\partial \rho Y_{i}}{\partial t}+\sum_{j=1}^{3} \frac{\partial \rho u_{j} Y_{i}}{\partial x_{j}}=-\sum_{j=1}^{3} \frac{\partial J_{i j}}{\partial x_{j}}+\rho \dot{\omega}_{i}(\mathbf{Y}, N), \quad i=1, \ldots, n_{s}
$$


where $J_{i j}(\mathbf{x}, t)$ denotes the diffusive flux of scalar $i$ along the $j$ th coordinate direction,

$$
J_{i j}(\mathbf{x}, t)=-\rho(\mathbf{x}, t) D(\mathbf{Y}(\mathbf{x}, t)) \frac{\partial Y_{i}(\mathbf{x}, t)}{\partial x_{j}},
$$

$D(\mathbf{Y}(\mathbf{x}, t))$ is the kinematic diffusivity of any scalar into the mixture and $\dot{\omega}_{i}(\mathbf{Y}(\mathbf{x}, t), N(\cdot, \mathbf{x}, t))$ represent the scalar production/destruction terms. The diffusivity $D(\mathbf{Y}(\mathbf{x}, t))$ can be related to the kinematic viscosity $\nu(\mathbf{Y}(\mathbf{x}, t))$ via a molecular Schmidt/Prandtl number $S c=0.7, D(\mathbf{Y}(\mathbf{x}, t))=\nu(\mathbf{Y}(\mathbf{x}, t)) / S c$ $[30,32]$. Frequently, the reactive scalars are taken as species mass fractions complemented by a calorific variable such as enthalpy. In the argument list of $\dot{\omega}_{i}$, the dependency on $N(\cdot, \mathbf{x}, t)$ indicates that the scalar source terms may be functionals of the particle size distribution (see below), for instance, owing to species consumption or release on account of particle formation.

In combusting flows at low Mach numbers, the mixture density $\rho(\mathbf{x}, t)$ is often computed in terms of the reactive scalars $\mathbf{Y}(\mathbf{x}, t)$,

$$
\rho(\mathbf{x}, t)=\hat{\rho}(\mathbf{Y}(\mathbf{x}, t)) .
$$

Physically, this implies that the impact of local pressure deviations from the nominal ambient pressure on the density is comparably small. With regard to its constitutive relations, moreover, the carrier fluid is considered as a multicomponent ideal gas.

For a particulate phase that is polydispersed with respect to a characteristic particle size $l \in[0, \infty)$, we consider, as basis for a Eulerian continuum formulation, the number density of particles $N(l, \mathbf{x}, t)$ per unit of mixture volume and per unit of length in particle size space. Following Hulburt and Katz [27], the evolution of the number density $N(l, \mathbf{x}, t)$ both in physical and in particle size space is governed by the population balance equation $(\mathrm{PBE})$

$$
\frac{\partial N}{\partial t}+\sum_{j=1}^{3} \frac{\partial u_{j} N}{\partial x_{j}}+\frac{\partial G(l, \mathbf{Y}) N}{\partial l}=-\sum_{j=1}^{3} \frac{\partial K_{j}}{\partial x_{j}}+\dot{s}(l, \mathbf{Y}, N)
$$

where $K_{j}(l, \mathbf{x}, t)$ represents the diffusive flux of number density along the $j$ th coordinate direction in physical space,

$$
K_{j}(l, \mathbf{x}, t)=-D_{p}(\mathbf{x}, t) \frac{\partial N(l, \mathbf{x}, t)}{\partial x_{j}},
$$

and $D_{p}(\mathbf{x}, t)$ denotes the common kinematic diffusivity of all particles. In view of the soot particle kinetics of Section 4.2, we consider $l$ as the diameter of a spherical particle. In this case, $G(l, \mathbf{Y}(\mathbf{x}, t))$ denotes the particle growth rate, while $\dot{s}(l, \mathbf{Y}(\mathbf{x}, t), N(\cdot, \mathbf{x}, t))$ encompasses the particle production and destruction rates due to nucleation and coalescence.

Frequently, the release or depletion of gas phase species briefly mentioned above is quantified with the aid of the moments of the particle size distribution $N(\cdot, \mathbf{x}, t)$,

$$
M_{k}(N(\cdot, \mathbf{x}, t))=\int_{0}^{\infty} l^{k} N(l, \mathbf{x}, t) d l .
$$

In view of subsequent developments, we restrict the semi-infinite particle size space $[0, \infty)$ to the finite domain $\left[l_{l}, L\right]$, where $l_{l}$ and $L$ represent, respectively, the minimum and maximum attainable particle diameters.

In variable density flows, it is advantageous to switch to a mass-based definition of the number density [5] and to consider the number of particles $N_{\rho}(l, \mathbf{x}, t)$ per unit of mixture mass and unit of length in particle size space,

$$
N_{\rho}(l, \mathbf{x}, t) \equiv \frac{N(l, \mathbf{x}, t)}{\rho(\mathbf{x}, t)} .
$$

For future reference, we record the initial conditions on the fluid composition and the mass-based particle number density,

$$
\begin{aligned}
\mathbf{Y}\left(\mathbf{x}, t_{0}\right) & =\mathbf{Y}_{0}(\mathbf{x}), \\
N_{\rho}\left(l, \mathbf{x}, t_{0}\right) & =N_{\rho, 0}(l, \mathbf{x}) .
\end{aligned}
$$




\subsection{Large eddy simulation}

Frequently, the LES-operator is introduced as a spatial filter acting on the governing fields. Since, for our developments, the precise definition (or construction) of such a filter is immaterial, we first recall that the LES-operator, ${ }^{-}$, implements an expectation operation and can thus be formulated in the following way [59]

$$
\bar{\Phi}\left(\mathbf{u}(\mathbf{x}, t), p(\mathbf{x}, t), \mathbf{Y}(\mathbf{x}, t), N_{\rho}(\cdot, \mathbf{x}, t)\right)=\int \Phi(\mathbf{v}, q, \mathbf{y}, n(\cdot)) f_{\mathbf{u}, p, \mathbf{Y}, N_{\rho}}(\mathbf{v}, q, \mathbf{y}, n(\cdot) ; \mathbf{x}, t) d \mathbf{v} d q d \mathbf{y} d n(\cdot),
$$

where $\Phi\left(\mathbf{u}(\mathbf{x}, t), p(\mathbf{x}, t), \mathbf{Y}(\mathbf{x}, t), N_{\rho}(\cdot, \mathbf{x}, t)\right)$ denotes a general observable that appears, formally, as a function of the local velocity, pressure and fluid composition and as a functional of the particle size distribution $N_{\rho}(\cdot, \mathbf{x}, t) . f_{\mathbf{u}, p, \mathbf{Y}, N_{\rho}}(\mathbf{v}, q, \mathbf{y}, n(\cdot) ; \mathbf{x}, t)$ is termed the LES-filtered $p d f$ associated with a single realization of the governing fields and may be related to a filter kernel $G\left(\mathbf{x}, \mathbf{x}^{\prime}\right)$ according to

$$
\begin{aligned}
f_{\mathbf{u}, p, \mathbf{Y}, N_{\rho}}(\mathbf{v}, q, \mathbf{y}, n(\cdot) ; \mathbf{x}, t)= & \overline{\delta(\mathbf{v}-\mathbf{u}(\mathbf{x}, t)) \delta(q-p(\mathbf{x}, t)) \delta(\mathbf{y}-\mathbf{Y}(\mathbf{x}, t)) \delta\left(n(\cdot)-N_{\rho}(\cdot, \mathbf{x}, t)\right)} \\
= & \int_{\Omega} G\left(\mathbf{x}, \mathbf{x}^{\prime}\right) \delta\left(\mathbf{v}-\mathbf{u}\left(\mathbf{x}^{\prime}, t\right)\right) \delta\left(q-p\left(\mathbf{x}^{\prime}, t\right)\right) \delta\left(\mathbf{y}-\mathbf{Y}\left(\mathbf{x}^{\prime}, t\right)\right) \\
& \times \delta\left(n(\cdot)-N_{\rho}\left(\cdot, \mathbf{x}^{\prime}, t\right)\right) d \mathbf{x}^{\prime},
\end{aligned}
$$

where $\delta(\cdot)$ indicates the Dirac delta distribution and $G(\mathbf{x}, \cdot) \geq 0$ integrates to unity. In Eqs. (13) and $(14), \mathbf{v}, q$ and $\mathbf{y}$ represent the sample space variables related to $\mathbf{u}(\mathbf{x}, t), p(\mathbf{x}, t)$ and $\mathbf{Y}(\mathbf{x}, t)$, respectively, while $n(\cdot)$ indicates the sample space function associated with the particle size distribution $N_{\rho}(\cdot, \mathbf{x}, t)$ at $(\mathbf{x}, t)$. Specifically, $n(\cdot)$ indicates a particular function drawn from the space of admissible particle size distributions. For notational clarity and to distinguish the nature of $n(\cdot)$ from that of the remaining sample space variables, we maintain the parenthesis-notation $n(\cdot)$ in the following developments. The integral in Eq. (13) is written over the combined sample space $(\mathbf{v}, q, \mathbf{y}, n(\cdot))$.

Conceptually, the marginal number density $p d f f_{N_{\rho}}(n(\cdot) ; \mathbf{x}, t)$ may be obtained as the limit functional

$$
f_{N_{\rho}}(n(\cdot) ; \mathbf{x}, t)=\overline{\lim _{m \rightarrow \infty} \prod_{i=0}^{m} \delta\left(n\left(l_{i}\right)-N_{\rho}\left(l_{i}, \mathbf{x}, t\right)\right)},
$$

where $l_{i}=l_{l}+i\left(L-l_{l}\right) / m, i=0, \ldots, m$, represents an auxiliary grid in particle size space and $n\left(l_{i}\right)$ are auxiliary sample space variables associated with $N_{\rho}\left(l_{i}, \mathbf{x}, t\right)$. Eq. (15) emphasizes that $f_{N_{\rho}}$ can be viewed as a multi-size, albeit one-point, one-time $p d f$ [65].

For subsequent developments, we record the following commutation property of the LES-operator

$$
\begin{aligned}
& \frac{\overline{\partial \mathbf{u}(\mathbf{x}, t)}}{\partial t}=\frac{\partial \overline{\mathbf{u}}(\mathbf{x}, t)}{\partial t}, \\
& \frac{\overline{\partial \mathbf{u}(\mathbf{x}, t)}}{\partial x_{i}}=\frac{\partial \overline{\mathbf{u}}(\mathbf{x}, t)}{\partial x_{i}}, \quad i=1, \ldots, 3,
\end{aligned}
$$

and similarly for the remaining governing fields $p(\mathbf{x}, t), \mathbf{Y}(\mathbf{x}, t)$ and $N_{\rho}(l, \mathbf{x}, t)$.

Based on Eqs. (6) and (13), the LES-filtered density field is given by

$$
\bar{\rho}(\mathbf{x}, t)=\overline{\hat{\rho}(\mathbf{Y}(\mathbf{x}, t))}=\int \hat{\rho}(\mathbf{y}) f_{\mathbf{Y}}(\mathbf{y} ; \mathbf{x}, t) d \mathbf{y}
$$

where $f_{\mathbf{Y}}(\mathbf{y} ; \mathbf{x}, t)$ represents the marginal filtered $p d f$ associated with a single realization of the reactive scalar fields. With the aid of Eq. (18), we further introduce the density weighted Favre-filter

$$
\tilde{\Phi}\left(\mathbf{u}(\mathbf{x}, t), p(\mathbf{x}, t), \mathbf{Y}(\mathbf{x}, t), N_{\rho}(\cdot, \mathbf{x}, t)\right)=\frac{\overline{\rho(\mathbf{x}, t) \Phi\left(\mathbf{u}(\mathbf{x}, t), p(\mathbf{x}, t), \mathbf{Y}(\mathbf{x}, t), N_{\rho}(\cdot, \mathbf{x}, t)\right)}}{\bar{\rho}(\mathbf{x}, t)} .
$$

Applying the LES-operator - to the continuity and momentum equations (Eqs. (1) and (2)) and taking into account Eq. (19) leads to the following governing LES equations [33]

$$
\begin{gathered}
\frac{\partial \bar{\rho}}{\partial t}+\sum_{j=1}^{3} \frac{\partial \bar{\rho} \tilde{u}_{j}}{\partial x_{j}}=0 \\
\frac{\partial \bar{\rho} \tilde{u}_{i}}{\partial t}+\sum_{j=1}^{3} \frac{\partial \bar{\rho} \tilde{u}_{i} \tilde{u}_{j}}{\partial x_{j}}=-\frac{\partial \bar{p}}{\partial x_{i}}+\sum_{j=1}^{3} \frac{\partial\left(\tilde{\tau}_{i j}-\tau_{i j}^{*}\right)}{\partial x_{j}}+\bar{\rho} g_{i}, \quad i=1, \ldots, 3,
\end{gathered}
$$


where $\tilde{\tau}_{i j}$ denotes the viscous stress tensor associated with the Favre-filtered velocity field $\tilde{\mathbf{u}}(\mathbf{x}, t)$ and $\tau_{i j}^{*}=\bar{\rho}\left(\widetilde{u_{i} u_{j}}-\tilde{u}_{i} \tilde{u}_{j}\right)$ is the residual stress tensor. Following common practice, we adopt the standard Smagorinsky model for the deviatoric component of $\tau_{i j}^{*}[32]$, while its spherical component is absorbed into the LES-filtered pressure $\bar{p}(\mathbf{x}, t)$.

\subsection{The joint scalar-number density $p d f$}

As a basis for modelling the evolution of the gas phase composition and the particle size distribution in the context of LES, we obtain, in this section, a transport equation for the joint scalar-number density $p d f,^{1}$

$$
f(\mathbf{y}, n(\cdot) ; \mathbf{x}, t)=\overline{g(\mathbf{y}, n(\cdot) ; \mathbf{x}, t)}=\overline{\delta(\mathbf{y}-\mathbf{Y}(\mathbf{x}, t)) \delta\left(n(\cdot)-N_{\rho}(\cdot, \mathbf{x}, t)\right)},
$$

in which the physical processes related to gas phase chemistry and particle formation appear naturally closed. Here, $g(\mathbf{y}, n(\cdot) ; \mathbf{x}, t)=\delta(\mathbf{y}-\mathbf{Y}(\mathbf{x}, t)) \delta\left(n(\cdot)-N_{\rho}(\cdot, \mathbf{x}, t)\right)$ represents the fine-grained density associated with $f(\mathbf{y}, n(\cdot) ; \mathbf{x}, t)$. For future reference, we also introduce the density weighted $p d f \tilde{f}(\mathbf{y}, n(\cdot) ; \mathbf{x}, t)$,

$$
\bar{\rho}(\mathbf{x}, t) \tilde{f}(\mathbf{y}, n(\cdot) ; \mathbf{x}, t) \equiv \hat{\rho}(\mathbf{y}) f(\mathbf{y}, n(\cdot) ; \mathbf{x}, t) .
$$

By following a standard procedure, we obtain on account of Eqs. (1), (4), (7) and (10) the following evolution equation for the density weighted fine-grained $p d f \rho g$

$$
\frac{\partial \rho g}{\partial t}+\sum_{j=1}^{3} \frac{\partial \rho u_{j} g}{\partial x_{j}}=-\sum_{i=1}^{n_{s}} \frac{\partial g}{\partial y_{i}}\left(\rho \dot{\omega}_{i}-\sum_{j=1}^{3} \frac{\partial J_{i j}}{\partial x_{j}}\right)-\frac{\partial g}{\partial n}\left(\dot{s}-\frac{\partial G(\cdot, \mathbf{Y}) \rho N_{\rho}}{\partial l}-\sum_{j=1}^{3} \frac{\partial K_{j}}{\partial x_{j}}\right)
$$

where arguments have been omitted for brevity. Recall, at this point, that any scalar functional $\Psi(\mathbf{u}, p$, $\left.\mathbf{Y}, N_{\rho}, \partial \mathbf{Y} / \partial \mathbf{x}, \ldots\right)$ of $\mathbf{u}(\mathbf{x}, t), p(\mathbf{x}, t), \mathbf{Y}(\mathbf{x}, t), N_{\rho}(\cdot, \mathbf{x}, t)$ and their spatial derivatives obeys the following identity [69]

$$
\overline{g \Psi\left(\mathbf{u}, p, \mathbf{Y}, N_{\rho}, \frac{\partial \mathbf{Y}}{\partial \mathbf{x}}, \ldots\right)}=\overline{f\left(\Psi\left(\mathbf{u}, p, \mathbf{Y}, N_{\rho}, \frac{\partial \mathbf{Y}}{\partial \mathbf{x}}, \ldots\right) \mid \mathbf{y}, n(\cdot)\right)},
$$

where the vertical bar indicates conditioning on the events $\mathbf{Y}(\mathbf{x}, t)=\mathbf{y}$ and $N_{\rho}(\cdot, \mathbf{x}, t)=n(\cdot)$.

With the aid of Eq. (25), the LES-filtered value of $\overline{\left(\rho u_{j} g\right)}$ can be reformulated according to

$$
\overline{\left(\rho u_{j} g\right)}=\hat{\rho}(\mathbf{y}) \overline{\left(u_{j} \mid \mathbf{y}, n(\cdot)\right)} f=\hat{\rho}(\mathbf{y}) \tilde{u}_{j} f-\hat{\rho}(\mathbf{y})\left(\tilde{u}_{j}-\overline{\left(u_{j} \mid \mathbf{y}, n(\cdot)\right)}\right) f .
$$

The turbulent transport term in Eq. (26) is commonly modelled by adopting a gradient diffusion hypothesis

$$
\hat{\rho}(\mathbf{y})\left(\tilde{u}_{j}-\overline{\left(u_{j} \mid \mathbf{y}, n(\cdot)\right)}\right) f=\bar{\rho}(\mathbf{x}, t) \Gamma(\mathbf{x}, t) \frac{\partial \tilde{f}}{\partial x_{j}} .
$$

Here, $\Gamma(\mathbf{x}, t)=\Gamma^{\prime}(\mathbf{x}, t) / S c^{\prime}$ represents a scaled eddy viscosity, $\Gamma^{\prime}(\mathbf{x}, t)$ denotes the eddy viscosity computed from the standard Smagorinsky model [38] and $S c^{\prime}=0.7$ is a constant turbulent Schmidt/Prandtl number.

By applying the LES operator to Eq. (24) and taking into account Eqs. (20), (23), (25) and (27) as well as the commutation property in Eqs. (16) and (17), we arrive at the following transport equation for the joint scalar-number density $p d f$

$$
\bar{\rho} \frac{\partial \tilde{f}}{\partial t}+\sum_{j=1}^{3} \bar{\rho} \tilde{u}_{j} \frac{\partial \tilde{f}}{\partial x_{j}}=\sum_{j=1}^{3} \frac{\partial}{\partial x_{j}}\left(\bar{\rho} \Gamma \frac{\partial \tilde{f}}{\partial x_{j}}\right)-\sum_{i=1}^{n_{s}+1} \frac{\partial}{\partial z_{i}}\left(\bar{\rho} s_{i}(\cdot, \mathbf{z}) \tilde{f}+\bar{\rho} \mathcal{M}_{i} \tilde{f}\right)
$$

where $\mathbf{z}=\left(\mathbf{y}^{T}, n(\cdot)\right)^{T}$ represents the joint scalar-number density sample space vector, $\mathcal{M}_{i} \tilde{f}$ encompasses the (unclosed) micromixing contribution

$$
\mathcal{M}_{i} \tilde{f}=-\frac{\tilde{f}}{\hat{\rho}(\mathbf{y})} \begin{cases}\sum_{j=1}^{3} \overline{\left(\frac{\partial J_{i j}}{\partial x_{j}} \mid \mathbf{y}, n(\cdot)\right)} & \text { for } i=1, \ldots, n_{s} \\ \sum_{j=1}^{3} \overline{\left(\frac{\partial K_{j}}{\partial x_{j}} \mid \mathbf{y}, n(\cdot)\right)} & \text { otherwise }\end{cases}
$$

\footnotetext{
${ }^{1}$ If instead we based the definition of $f(\mathbf{y}, n(\cdot) ; \mathbf{x}, t)$ on the volumetric number density $N(\cdot, \mathbf{x}, t)$, then the transport equation for $\tilde{f}$ would include an additional unclosed term involving $\overline{\left(\sum_{j=1}^{3} \frac{\partial u_{j}}{\partial x_{j}} \mid \mathbf{y}, n(\cdot)\right)}$.
} 
and the vector-valued source term $\mathbf{s}(\cdot, \mathbf{z})$ is given by

$$
\mathbf{s}(\cdot, \mathbf{z})=\left(\begin{array}{c}
\dot{\boldsymbol{\omega}}(\mathbf{y}, n(\cdot)) \\
\frac{1}{\hat{\rho}(\mathbf{y})}\left(\dot{s}(\cdot, \mathbf{y}, \hat{\rho}(\mathbf{y}) n(\cdot))-\frac{\partial(G(\cdot, \mathbf{y}) \hat{\rho}(\mathbf{y}) n(\cdot))}{\partial l}\right)
\end{array}\right) .
$$

\subsection{Micromixing model}

In order to close the molecular mixing term in Eq. $(29)_{1}$, we adopt the model proposed by McDermott and Pope [42]. Specifically, these authors augmented the IEM micromixing model for relaxing a scalar $Y_{i}(\mathbf{x}, t), i=1, \ldots, n_{s}$, towards its Favre-filtered counterpart $\tilde{Y}_{i}(\mathbf{x}, t)$ by a spatially diffusive transport of $\tilde{Y}_{i}(\mathbf{x}, t)$,

$$
\mathcal{M}_{i} \tilde{f} \equiv m_{i}(\mathbf{x}, t, \mathbf{z}) \tilde{f}=\left[\kappa(\mathbf{x}, t)\left(\tilde{Y}_{i}-y_{i}\right)+\frac{1}{\bar{\rho}} \sum_{j=1}^{3} \frac{\partial}{\partial x_{j}}\left(\bar{\rho} \tilde{D} \frac{\partial \tilde{Y}_{i}}{\partial x_{j}}\right)\right] \tilde{f}
$$

where $\kappa(\mathbf{x}, t)$ represents a micromixing frequency common to all scalars,

$$
\kappa(\mathbf{x}, t)=\frac{C_{\kappa}}{2} \frac{\Gamma(\mathbf{x}, t)}{\Delta^{2}}
$$

and $\Delta$ is obtained as the cubic root of the local cell volume for a finite volume based spatial discretization scheme. The micromixing model in Eq. (31) may similarly be formulated for differential diffusion among gas phase scalars and consistently reduces to a pure diffusion term in the limit as the LES operator approaches the identity and a direct numerical simulation (DNS) is recovered. By adapting Eq. (31) to the number density micromixing term in Eq. $(29)_{2}$, we further obtain the following expression for $\mathcal{M}_{n_{s}+1} \tilde{f}$

$$
\mathcal{M}_{n_{s}+1} \tilde{f} \equiv m_{n_{s}+1}(\mathbf{x}, t, \mathbf{z}) \tilde{f}=\left[\kappa(\mathbf{x}, t)\left(\tilde{N}_{\rho}-n(\cdot)\right)+\frac{1}{\bar{\rho}} \sum_{j=1}^{3} \frac{\partial}{\partial x_{j}}\left(\tilde{D}_{p} \frac{\partial \bar{\rho} \tilde{N}_{\rho}}{\partial x_{j}}\right)\right] \tilde{f}
$$

Jointly, the final terms in Eqs. (31) and (33) account for differential diffusion between the gas and particulate phases.

For the turbulent, non-premixed flame analyzed in Sections 5 through 7 , the particle diffusivity $D_{p}$ is set to zero identically. Strictly, by Eq. (29) 2 , this implies $\mathcal{M}_{n_{s}+1} \tilde{f}=0$ which is at variance with the modelled micromixing term $\mathcal{M}_{n_{s}+1} \tilde{f}=m_{n_{s}+1} \tilde{f}=\kappa(\mathbf{x}, t)\left(\bar{N}_{\rho}(\cdot, \mathbf{x}, t)-n(\cdot)\right)$ obtained from Eq. (33). Thus, even in the absence of molecular particle diffusion, the present micromixing model accounts for mixing of number density towards the mean at a rate that is proportional to the local scaled eddy viscosity $\Gamma(\mathbf{x}, t)$. One possible approach to resolve this contradiction would be to choose different micromixing frequencies (variants of Eq. (32)) for the scalars and number density and to express the individual micromixing frequencies in terms of both $\Gamma(\mathbf{x}, t)$ and the respective molecular diffusivity such that the right hand sides of Eqs. (31) and (33) consistently reduce to zero in the limit of vanishing molecular diffusion. This extension may also yield differential micromixing frequencies among the gas phase scalars. In view of the scope of this article, we defer such a model enhancement to future times, keeping, however, the limitations of the present micromixing closure in mind.

\subsection{The stochastic field equations}

In Sections 3.3 and 3.4, we obtained the joint scalar-number density $p d f$ transport equation (Eq. (28) and Eqs. (31), (33)) as a model for the evolution of a fluid and an immersed particulate phase in a given flow field $\tilde{\mathbf{u}}(\mathbf{x}, t)$. Due to the large number of independent variables in Eq. (28), direct numerical discretization techniques may entail a prohibitive computational expense. At the same time, from a physical viewpoint, our objective is not to accurately compute $\tilde{f}(\mathbf{z} ; \mathbf{x}, t)$, but rather to approximate low order expectations with respect to $\tilde{f}(\mathbf{z} ; \mathbf{x}, t)$ such as the Favre-filtered reactive scalars $\tilde{\mathbf{Y}}(\mathbf{x}, t)$ and the Favre-filtered particle size distribution $\tilde{N}_{\rho}(\cdot, \mathbf{x}, t)$,

$$
\left(\begin{array}{c}
\tilde{\mathbf{Y}}(\mathbf{x}, t) \\
\tilde{N}_{\rho}(\cdot, \mathbf{x}, t)
\end{array}\right)=\int \mathbf{z} \tilde{f}(\mathbf{z} ; \mathbf{x}, t) d \mathbf{z}
$$


Within the combustion community, this has motivated the development of solution approaches based on an independent stochastic process $\boldsymbol{\theta}(t ; l, \mathbf{x})$ which is constructed in such a way that the transition $p d f$ $h\left(\mathbf{z}, t \mid \mathbf{Y}_{0}(\mathbf{x}), N_{\rho, 0}(\cdot, \mathbf{x}), t_{0} ; \mathbf{x}\right)$ associated with $\boldsymbol{\theta}(t ; \cdot, \mathbf{x})$ obeys Eq. (28) subject to the initial condition

$$
h\left(\mathbf{z}, t_{0} \mid \mathbf{Y}_{0}(\mathbf{x}), N_{\rho, 0}(\cdot, \mathbf{x}), t_{0} ; \mathbf{x}\right)=\delta\left(\mathbf{y}-\mathbf{Y}_{0}(\mathbf{x})\right) \delta\left(n(\cdot)-N_{\rho, 0}(\cdot, \mathbf{x})\right)
$$

Since both the initial time $t_{0}$ and the initial fields $\mathbf{Y}_{0}(\mathbf{x})$ and $N_{\rho, 0}(\cdot, \mathbf{x})$ are deterministic, we drop the conditioning on $\left(\mathbf{Y}_{0}(\mathbf{x}), N_{\rho, 0}(\cdot, \mathbf{x}), t_{0}\right)$ from the argument list of $h$ for clarity and write $h=h(\mathbf{z}, t ; \mathbf{x})$. In a Monte Carlo-type solution method, several realizations (samples) $\boldsymbol{\theta}^{(1)}(t ; l, \mathbf{x}), \ldots, \boldsymbol{\theta}^{\left(n_{f}\right)}(t ; l, \mathbf{x})$ of such a stochastic process $\boldsymbol{\theta}(t ; l, \mathbf{x})$ are computed and expectations are approximated by Monte Carlo estimates,

$$
\tilde{F}\left(\mathbf{Y}(\mathbf{x}, t), N_{\rho}(\cdot, \mathbf{x}, t)\right) \approx \frac{1}{n_{f}} \sum_{i=1}^{n_{f}} F\left(\boldsymbol{\theta}^{(i)}(t ; \cdot, \mathbf{x})\right)
$$

where $F\left(\mathbf{Y}, N_{\rho}\right)$ represents an observable expressed in terms of $\mathbf{Y}(\mathbf{x}, t)$ and $N_{\rho}(\cdot, \mathbf{x}, t)$. This approach channels the computational effort towards the accurate computation of low order moments of $h(\mathbf{z}, t ; \mathbf{x})=$ $\tilde{f}(\mathbf{z} ; \mathbf{x}, t)$, while the statistical error is accumulated on higher order moments.

In the present article, we specifically invoke the method of Eulerian stochastic fields to construct the stochastic process $\boldsymbol{\theta}(t ; l, \mathbf{x})$. This approach preserves the Eulerian nature of the physical model; it was originally developed by Valiño [72], Hauke and Valiño [26] and Sabel'nikov and Soulard [67]. Formally, the stochastic field equations are given by

$$
\begin{aligned}
\bar{\rho} \frac{\partial \theta_{i}}{\partial t}+\sum_{j=1}^{3} \bar{\rho} \tilde{u}_{j} \frac{\partial \theta_{i}}{\partial x_{j}}= & \sum_{j=1}^{3} \frac{\partial}{\partial x_{j}}\left(\bar{\rho} \Gamma(\mathbf{x}, t) \frac{\partial \theta_{i}}{\partial x_{j}}\right)-\bar{\rho} \sqrt{2 \Gamma(\mathbf{x}, t)} \sum_{j=1}^{3} \dot{W}_{j}(t) \frac{\partial \theta_{i}}{\partial x_{j}} \\
& +\bar{\rho}\left(s_{i}(l, \boldsymbol{\theta})+m_{i}(\mathbf{x}, t, \boldsymbol{\theta})\right), \quad i=1, \ldots, n_{s}+1
\end{aligned}
$$

and correspond to continuous-time stochastic processes which are smoothly parameterized by $(l, \mathbf{x})$. In Eq. (37), $\mathbf{W}(t)$ denotes a three-dimensional temporal Wiener process with (formal) time derivative $\mathbf{W}(t)$. Strictly, Eq. (37) is only formally valid, holding in a time-integral sense

$$
\int_{t_{0}}^{t} d \theta_{i}(t ; l, \mathbf{x})=\int_{t_{0}}^{t} \frac{\partial \theta_{i}}{\partial t} d t=-\sum_{j=1}^{3} \int_{t_{0}}^{t} \tilde{u}_{j} \frac{\partial \theta_{i}}{\partial x_{j}} d t+\ldots-\sum_{j=1}^{3} \int_{t_{0}}^{t} \sqrt{2 \Gamma(\mathbf{x}, t)} \frac{\partial \theta_{i}}{\partial x_{j}} d W_{j}(t)+\ldots
$$

where the stochastic integral with respect to $\mathbf{W}(t)$ is interpreted in Itô's sense. In Appendix A we show that if the stochastic fields $\boldsymbol{\theta}(t ; l, \mathbf{x})$ evolve according to Eq. (37) subject to the deterministic initial condition $\boldsymbol{\theta}\left(t_{0} ; l, \mathbf{x}\right)=\left(\mathbf{Y}_{0}(\mathbf{x}), N_{\rho, 0}(l, \mathbf{x})\right)$, then the transition $p d f h(\mathbf{z}, t ; \mathbf{x})$ associated with the stochastic fields evolves according to Eq. (28). (This proof generalizes the derivation of Sewerin and Rigopoulos [69, Appendix B] to variable density flows at low Mach number.)

\section{Gas phase and soot kinetics}

\subsection{Gas phase kinetics and radiation}

The gas phase chemical kinetics for methane combustion are based on the GRI 1.2 reaction mechanism $[18,19]$. In order to model radiation, we adopt the hypothesis of optical thinness. Following Lindstedt and Louloudi [39], the loss in enthalpy $\mathrm{H}(\mathbf{Y}, N)$ due to radiation from gas phase $\mathrm{H}_{2} \mathrm{O}, \mathrm{CO}_{2}, \mathrm{CH}_{4}$ and $\mathrm{CO}$ as well as from soot can be computed according to

$$
\dot{\omega}_{H}(\mathbf{Y}, N)=-\frac{4 \sigma}{\hat{\rho}(\mathbf{Y})}\left(T(\mathbf{Y})^{4}-T_{b}^{4}\right) \sum_{i=1}^{4} a_{p, i}(T(\mathbf{Y})) p_{i}(\mathbf{Y})-\frac{4 \sigma}{\hat{\rho}(\mathbf{Y})} C_{s} f_{v}(N)\left(T(\mathbf{Y})^{5}-T_{b}^{5}\right)
$$

where $\sigma$ denotes the Stefan-Boltzmann constant, $T(\mathbf{Y})$ indicates temperature, $i=\mathrm{H}_{2} \mathrm{O}, \mathrm{CO}_{2}, \mathrm{CH}_{4}, \mathrm{CO}$ represents an index running through the major radiating species, $p_{i}(\mathbf{Y})$ is the partial pressure of species $i$ (in $[\mathrm{atm}]$ ) and $a_{p, i}(T)$ denotes its corresponding Planck mean absorption coefficient. The latter is computed in terms of temperature from polynomial curve fit expressions of the RADCAL model [1, 22]. 
Furthermore, $T_{b} \equiv 295 \mathrm{~K}$ denotes the ambient background temperature, $C_{s} \equiv 1307 / \mathrm{m}-\mathrm{K}[39]$ and $f_{v}(N)=\pi / 6 M_{3}(N)$ is the soot particle volume fraction.

One implication of the optical thinness hypothesis is that no reabsorption of thermal radiation occurs within the flame. In heavily sooting flames this may lead to an overprediction of the radiative heat loss and, by consequence, to a local underprediction of temperature [70]. The Delft III flame, however, is only lightly sooting with measured soot volume fractions below $2.5 \mathrm{ppb}$.

\subsection{Soot kinetics}

In the present article, we focus on the kinetic processes of soot nucleation, growth and oxidation and invoke kinetic rate expressions which have previously been employed in the context of laminar methane and ethylene diffusion flames $[4,24,40,71]$. Since the coalescent growth of a soot particle from two parent particles may only be important in the initial stages of particle creation and since aggregation preserves the number density of primary soot particles [40], we omit both coalescence and aggregation and interpret $N(l, \mathbf{x}, t)$ as the primary soot particle number density. Formally, however, the univariate PBE is able to accommodate coagulation.

Following Liu et al. [40], the nucleation rate is controlled by the molar concentration of acetylene and can be computed from

$$
s_{N}(\mathbf{Y})=\frac{2 N_{A}}{C_{\min }}\left[\mathrm{C}_{2} \mathrm{H}_{2}\right] k_{1}(T),
$$

where $N_{A}$ denotes Avogadro's number, $C_{\min }=700$ is the number of carbon atoms in a soot nucleus, $k_{1}(T)=1.7 \exp (-7548 \mathrm{~K} / T)(1 / s)$ and square brackets indicate molar concentrations. The specific surface growth rate (in $\left[\mathrm{kg} / \mathrm{m}^{2}-\mathrm{s}\right]$ ) of primary soot particles, on the other hand, is given by

$$
s_{\mathrm{C}_{2} \mathrm{H}_{2}}(\mathbf{Y})=2 p_{\mathrm{C}_{2} \mathrm{H}_{2}}(\mathbf{Y}) k_{2}(T),
$$

where $p_{\mathrm{C}_{2} \mathrm{H}_{2}}(\mathbf{Y})$ represents the acetylene partial pressure (in [atm]) and $k_{2}(T)=470 \exp (-16004 \mathrm{~K} / T)(\mathrm{s} / \mathrm{m})$ $[24,71]$. The leading factor of 2 in Eq. (41) is purely empirical; it has been introduced by Smooke et al. [71] in order to bring the growth rate which has originally been determined based on measurements in a laminar premixed ethylene flame [25] closer to measurements taken in laminar diffusion flames. The mean soot nuclei size amounts to $l_{\text {nuc }}=2.5 \times 10^{-9} \mathrm{~m}$ and the minimum and maximum attainable particle sizes are set to $l_{l}=2.5 \times 10^{-10} \mathrm{~m}$ and $L=10^{-5} \mathrm{~m}$, respectively.

Additionally, primary soot particles may shrink on account of oxidative surface reactions with hydroxyl or molecular oxygen $[24,51]$. Expressed in terms of the primary particle mass, the respective specific shrinkage rates (in $\left[\mathrm{kg} / \mathrm{m}^{2}-\mathrm{s}\right]$ ) are computed according to

$$
\begin{aligned}
& s_{\mathrm{OH}}(\mathbf{Y})=167 \frac{p_{\mathrm{OH}}(\mathbf{Y})}{\sqrt{T}}, \\
& s_{\mathrm{O}_{2}}(\mathbf{Y})=1200 p_{\mathrm{O}_{2}}(\mathbf{Y})\left(\frac{K_{A}(T) \chi}{1+K_{z}(T) p_{\mathrm{O}_{2}}}+K_{B}(T) \chi^{\prime}\right),
\end{aligned}
$$

where $p_{\mathrm{OH}}(\mathbf{Y})$ and $p_{\mathrm{O}_{2}}(\mathbf{Y})$ denote the partial pressures (in [atm]) of hydroxyl and molecular oxygen, respectively, $\chi$ and $\chi^{\prime}$ represent fractions defined by

$$
\chi=\left(1+\frac{K_{T}(T)}{K_{B}(T) p_{\mathrm{O}_{2}}}\right)^{-1}, \quad \chi^{\prime}=1-\chi,
$$

and $K_{A}(T), K_{B}(T), K_{T}(T)$ and $K_{z}(T)$ are the temperature dependent parameters introduced by Nagle and Strickland-Constable [51, p. 162]. Finally, the cumulative primary particle growth/oxidation rate $G(\mathbf{Y})$ can be computed by summing Eqs. (41) through (43) and converting from a mass-based to a diameter-based rate expression

$$
G(\mathbf{Y})=\frac{2}{\rho_{s}}\left(s_{\mathrm{C}_{2} \mathrm{H}_{2}}(\mathbf{Y})-s_{\mathrm{OH}}(\mathbf{Y})-s_{\mathrm{O}_{2}}(\mathbf{Y})\right),
$$

where $\rho_{s}=1900 \mathrm{~kg} / \mathrm{m}^{3}$ denotes the density of soot [40].

If primary soot particles are created and grow or, conversely, shrink due to oxidation, then gas phase species are consumed or released. This can be taken into account by augmenting the source terms due to 
chemical reactions by rate expressions based on the soot formation and oxidation stoichiometry as well as Eqs. (40) through (43). For acetylene and molecular hydrogen, for instance, we have

$$
\frac{1}{M W_{\mathrm{C}_{2} \mathrm{H}_{2}}} \dot{\omega}_{\mathrm{C}_{2} \mathrm{H}_{2}}^{\star}(\mathbf{Y}, N)=-\frac{1}{M W_{\mathrm{H}_{2}}} \dot{\omega}_{\mathrm{H}_{2}}^{\star}(\mathbf{Y}, N)=-\frac{s_{\mathrm{C}_{2} \mathrm{H}_{2}}(\mathbf{Y}) f_{a}(N)}{2 \hat{\rho}(\mathbf{Y}) M W_{s}}-\frac{s_{N}(\mathbf{Y}) C_{\mathrm{min}}}{2 \hat{\rho}(\mathbf{Y}) N_{A}},
$$

where the superscript ${ }^{*}$ indicates that the respective scalar sink/source terms exclude contributions from the gas phase reaction mechanism. $M W_{i}$ denotes the molecular weight of species $i, M W_{s} \equiv$ $12.011 \mathrm{~kg} / \mathrm{kmol}$ represents the molecular weight of solid soot and $f_{a}(N)=\pi M_{2}(N)$ denotes the local soot particle surface fraction.

In view of the findings of References [5, 37, 52], we set the kinematic diffusivity of soot in Eq. (33) to zero, $D_{p}(\mathbf{x}, t) \equiv 0$ (also see Section 3.4).

\section{Delft III flame configuration}

The Delft III flame consists of a central fuel jet (Dutch natural gas) surrounded by two concentric co-flows of air at atmospheric pressure and an ambient temperature of $295 \mathrm{~K}$ [57]. The nozzle encompasses a wide cylindrical ring with inner diameter $d=6 \mathrm{~mm}$ and outer diameter $15 \mathrm{~mm}$ featuring twelve equidistant holes of diameter $0.5 \mathrm{~mm}$ on a circle with diameter $7 \mathrm{~mm}$ from which the pilot flames emanate. The nozzle is embraced by an annulus of outer diameter $45 \mathrm{~mm}$ for the primary air co-flow which is, in turn, surrounded by a secondary air co-flow. The jet flows at a bulk velocity of $21.9 \mathrm{~m} / \mathrm{s}(R e \approx 8370)$, while the bulk velocities of the primary and secondary air co-flows amount to $4.4 \mathrm{~m} / \mathrm{s}$ and $0.3 \mathrm{~m} / \mathrm{s}$, respectively. The Delft III flame is characterized by strong extinction and reignition in the nearfield and possesses a lightly sooting flame head. Figure 1 schematically illustrates the geometry of the Delft burner tip as well as our computational domain.

Following Merci et al. [43, 44], we take the fuel composition as a mixture of methane and nitrogen with the same calorific value as Dutch natural gas $\left(85.3 \% \mathrm{CH}_{4}\right.$ and $14.7 \% \mathrm{~N}_{2}$ by volume). The pilot flames, moreover, burn a mixture of hydrogen, acetylene and air with a $\mathrm{H}$ to $\mathrm{C}$ ratio of 4 and an equivalence ratio of $1.4\left(16.936 \% \mathrm{H}_{2}, 5.682 \% \mathrm{C}_{2} \mathrm{H}_{2}, 16.258 \% \mathrm{O}_{2}\right.$ and $61.124 \% \mathrm{~N}_{2}$ by volume). Here, air is assumed to consist of $21 \% \mathrm{O}_{2}$ and $79 \% \mathrm{~N}_{2}$ by volume. For simplicity, the twelve pilot flames are replaced by a concentric inflow through an annulus in the nozzle rim with inner diameter $8 \mathrm{~mm}$ and outer diameter $9 \mathrm{~mm}$ at the experimental pilot flame mass flow rate of $2.3 \times 10^{-5} \mathrm{~kg} / \mathrm{s}$. Note that contrary to Ayache and Mastorakos [6] and Dodoulas and Navarro-Martinez [13] the pilot stream is separated from the fuel jet by a wall of $1 \mathrm{~mm}$ thickness in order to avoid the pilot gases from diffusing into the fuel mixture upstream of the nozzle exit plane. Although this representation of the pilot underestimates the experimental pilot flames' momentum flow rate, we found it to be sufficient to ignite the flame and lead to flame attachment at the nozzle rim (as observed in the experiment). The pilot inflow composition is taken as the chemical equilibrium composition corresponding to the unburned pilot pre-mixture at $295 \mathrm{~K}$ and the pilot inflow temperature is set to $1900 \mathrm{~K}$.

Experimentally, the Delft III flame was first investigated by Peeters et al. [57] who reported measurements of velocity statistics, mean temperature as well as concentrations of $\mathrm{OH}$ and a passive scalar. Subsequently, additional measurements of the velocity field, concentrations of the major species and temperature statistics were obtained, see Nooren et al. [55] and references therein. Recently, Qamar et al. [60] augmented the experimental database of the Delft III flame by measurements of mean soot volume fraction, intermittency and centerline $p d f s$ of instantaneous soot volume fraction. These measurements were obtained in the downstream region of the flame $(x / d \gtrsim 50)$, where unfortunately no velocity, temperature or scalar measurements are available. As Mueller and Raman [49] pointed out, this may render drawing definitive conclusions from a comparison of model predictions with measured soot-related quantities difficult.

\section{$6 \quad$ Numerical solution scheme and implementational aspects}

The combined LES-PBE-PDF model for a turbulent combusting flow with soot formation was implemented in our in-house research software LES-BOFFIN [29]. The solution method is based on a second order accurate finite volume scheme on a staggered grid encompassing a standard SIMPLE loop for the 


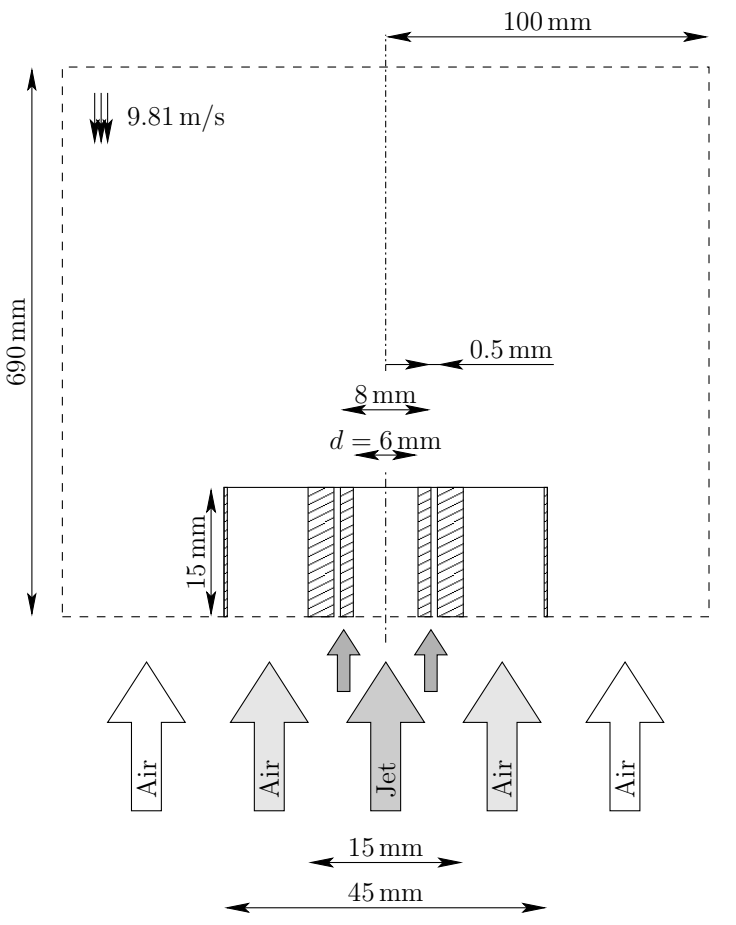

Figure 1: Schematic illustration of the Delft burner nozzle and the computational domain for the Delft III flame calculation.

coupled mass and momentum LES conservation equations (Eqs. (20) and (21)). As is common, the transport equations for the stochastic fields governing the gas composition (Eq. (37) for $i=1, \ldots, n_{s}$ ) are solved using a fractional steps method including in turns the processes of convection/diffusion, molecular mixing and gas phase reaction. Formally, the fractional time stepping constitutes a first order approximation in time [58]; its main benefit is that the numerical solution schemes can be tailored to the physical processes which they target.

The stochastic field equation associated with the particle number density, moreover, is discretized in particle size space using the explicit adaptive grid method recently developed by Sewerin and Rigopoulos [68] in combination with a high resolution finite volume method [36], also see Sewerin and Rigopoulos [69]. Here, the grid design is controlled by three parameters, the total number of nodes, the minimum node density in the nucleation interval and the maximum grid stretching, which we set to 30,4 nodes $/ 4.75 \mathrm{~nm}$ and 2 , respectively. Similar to the application of a fractional steps scheme to the stochastic equations governing the gas phase scalars, the stochastic field equation for the (transformed) number density is decomposed into a convection/diffusion, molecular mixing and PBE fractional step [68, 69].

In the convection/diffusion fractional step, the convective terms are discretized using a TVD stencil based on van Leer's limiter, while the remaining spatial derivatives are approximated by second order accurate central differences. For the temporal integration of the deterministic terms, the second order accurate Crank-Nicolson method is applied. The stochastic terms, by contrast, are integrated in time using an Euler-Maruyama scheme [56, Section 5.2]. During the reaction fractional step we employ the first order accurate implicit Euler method for temporally integrating the GRI 1.2 chemical kinetics, while the explicit 5th order accurate Runge-Kutta method DOPRI5 [23] is used for integrating in time the particle phase kinetics. For efficiency, the gas phase reaction fractional step is only executed for fluid cells whose temperature exceeds $800 \mathrm{~K}$. The PBE fractional step, on the other hand, is solved for all fluid cells. In order to further accelerate the solver for the reaction fractional step, we hard-coded the instructions for evaluating the reaction rates of the GRI 1.2 reaction mechanism, implemented a facility for computing the temperature-dependent kinetic coefficients only once per time step and grid point, and adopted a modified Newton-Raphson scheme for solving the backward Euler non-linear system. Cumulatively, these measures yield a reduction in runtime of the reaction step by about one order of magnitude.

In line with previous applications of the stochastic field method in the context of LES-PDF models [31- 
33], we invoke eight realizations of the stochastic fields. Mustata et al. [50], specifically, compared solutions computed using both eight and sixteen stochastic fields and observed very small differences between the resulting predictions of temporal mean and root mean square (rms) profiles for selected reactive scalars. However, this agreement may have been, in part, due to the accurate spatial resolution in their LES, entailing a low level of residual fluctuations. Subsequently, Jones and Navarro-Martinez [30] investigated autoignition in a lifted turbulent $\mathrm{H}_{2}$-air flame and also found eight and sixteen stochastic fields to yield almost coincidental profiles of mean and rms temperature, although the rms predictions slightly improved for sixteen stochastic fields. These authors additionally estimated the instantaneous finite sampling error for eight stochastic fields as approximately $10 \%$, pointing out that this error significantly reduces on time averaging.

The computational domain is cylindrical in shape and spans $16.67 d$ in the radial direction and $115 d$ in the axial direction, where $d=6 \mathrm{~mm}$ denotes the nozzle diameter (Figure 1). Since the wide nozzle rim of the Delft III burner head acts as a bluff body enhancing the mixing and reinforcing the shear layer in the near-field, we include a representation of the burner nozzle which extends by $2.5 d$ into the domain. The finite volume grid encompasses 672,70 and 36 cells in the axial, radial and circumferential directions, respectively. Axially, the grid is stretched by a factor of 1.002 and, radially, cells are thinner near the inner and outer nozzle diameter and stretch by a factor of 1.04 towards the lateral domain boundaries. In the circumferential direction, by contrast, a uniform grid is employed.

The mean axial velocity inflow profiles of both the fuel jet and the primary air co-flow are taken as power law profiles with exponents $1 / 6$ and $1 / 2$, respectively. The secondary air co-flow, on the other hand, features a constant mean axial velocity inflow profile. The velocity turbulence intensities for the jet and primary air co-flow, moreover, are set to $10 \%$, while the turbulence intensity in the secondary co-flow amounts to $1 \%$. The axial and radial rms inflow velocities vary quadratically within the jet (at the nozzle rim, they exceed the nominal centerline value by a factor of four) and reduce to constant profiles in the primary and secondary air co-flows, respectively. Along the lateral boundaries of the domain, both the stochastic scalars and the velocity field are subject to Dirichlet boundary conditions based on nominal values in the secondary co-flow. Finally, at the domain outlet, zero-gradient and convective outflow boundary conditions apply, respectively, to the stochastic scalars and the velocity field.

Temporal statistics were computed over a time period of approximately $250 \times 10^{-3} \mathrm{~s}$ and complemented by circumferential averaging. The time measurements which we provide were obtained on 4 nodes of a Cray XC30 Supercomputer (ARCHER UK) and averaged over 100 time steps of $1.2 \times 10^{-6} \mathrm{~s}$ at a point in time at which the temporal statistics of temperature had become time invariant.

\section{Results and discussion}

In order to assess the validity of our inflow boundary conditions, the time averaged (mean) and rms profiles of axial velocity ${ }^{2}$ are compared with the experimentally measured profiles at $3 \mathrm{~mm}$ above the nozzle exit plane in Figure 2. Except for a slight overprediction on the centerline, our choice of inflow boundary conditions approximates well the experimental mean axial velocity profile, including the weak recirculation zone above the nozzle rim. The rms of axial velocity are also well reproduced in the jet, except near the burner rim, but fall below the measured values in the primary co-flow, very similar to the rms profile obtained by Ayache and Mastorakos [6].

Figure 3 shows the radial profiles of the mean axial velocity and temperature at $50 \mathrm{~mm}, 150 \mathrm{~mm}$ and $250 \mathrm{~mm}$ above the nozzle exit plane as well as the corresponding measurements from the Delft III database. At the first two measurement stations, the mean axial velocity agrees well with the measured values, while it is slightly underpredicted further downstream, indicating that the jet spreads rather too rapidly. The mean temperature, moreover, is slightly overpredicted on the lean side of the reaction zone and the maximum mean temperature exceeds the measured maximum value by approximately $300 \mathrm{~K}$. Since this overprediction is passed downstream, its main cause seems to persist in the near-nozzle region and may be related to the spatial resolution of our LES in the nearfield as well as the effectiveness or accuracy of the eddy viscosity and micromixing closures. Indeed, other LES-based investigations of the Delft III flame $[6,13,48]$ reported much better agreement for the nearfield predictions using grids whose radial mesh spacing was smaller than the one in our grid by up to a factor of three.

\footnotetext{
${ }^{2}$ For conciseness, we omit the term 'Favre-filtered' when referring to both instantaneous and time averaged Favre-filtered variables.
} 

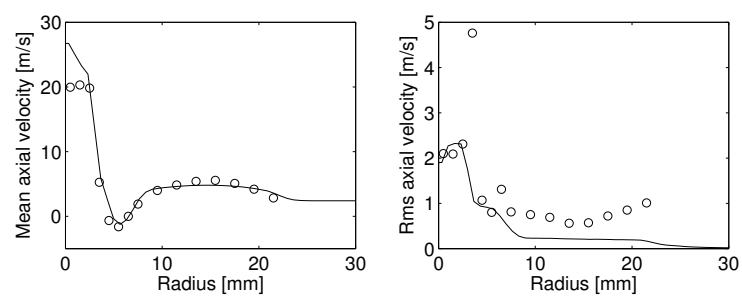

Figure 2: Comparing radial profiles of the mean and rms axial velocity (lines) with experimental measurements (symbols) at $3 \mathrm{~mm}$ above the nozzle exit plane.
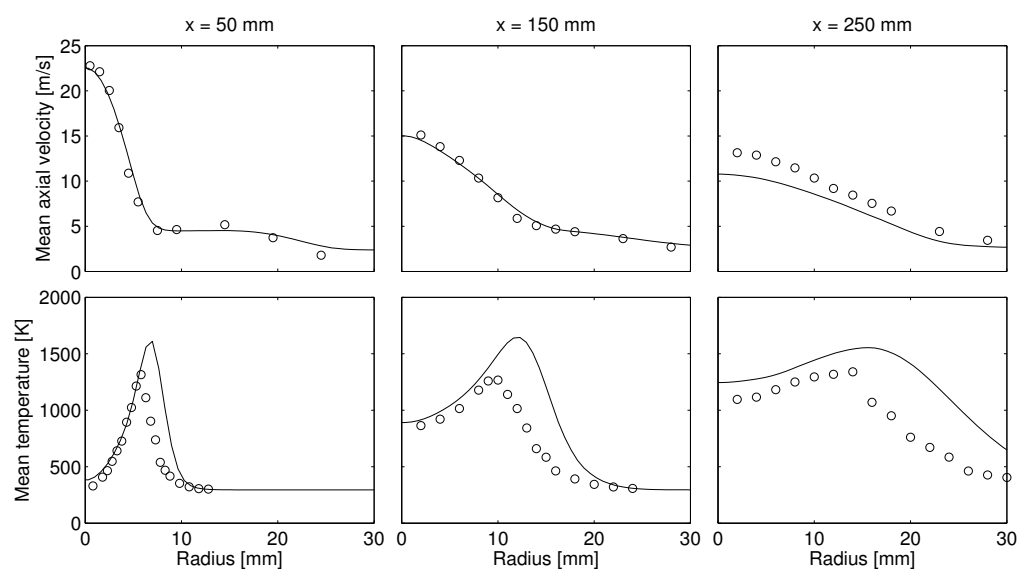

Figure 3: Comparing radial profiles of the mean axial velocity and temperature (lines) with experimental measurements (symbols) at $50 \mathrm{~mm}, 150 \mathrm{~mm}$ and $250 \mathrm{~mm}$ above the nozzle exit plane.

For the same axial measurement stations as in Figure 3, Figure 4 depicts both predicted and measured radial profiles of the mean mass fractions of $\mathrm{CO}_{2}, \mathrm{H}_{2} \mathrm{O}, \mathrm{H}_{2}$ and $\mathrm{CO}$. The agreement here reflects the discrepancy which we observed above for the temperature profiles: At the first measurement station, the gas composition is well reproduced on the rich side, while the maximum mass fractions and the values on the lean side are slightly overpredicted. The centerline value of $\mathrm{CO}_{2}$ is slightly underpredicted at $x=150 \mathrm{~mm}$, while the CO mass fraction is overpredicted near the centerline, the overprediction persisting throughout the radial profile. In general, the species profiles seem to be shifted radially outwards, reflecting the slightly excessive jet spreading. This is even more severe at the furthest measurement station, where the species profiles appear to be very diffusive. Here, the mass fractions of $\mathrm{H}_{2} \mathrm{O}$ and $\mathrm{CO}$ are overpredicted throughout and the $\mathrm{H}_{2}$ mass fraction is notably underpredicted in the jet core.

Figure 5 depicts contour plots of the mean temperature, soot number density and soot volume fraction. The superimposed contour in the left panel indicates the stoichiometric mixture fraction iso-line. For the center and right panels, the soot number and volume densities were computed as the zeroth and third moments, respectively, of the LES-filtered soot size distributions. At least qualitatively, the contours of the mean soot number density and volume fraction are in line with those reported by Mueller and Pitsch [48]. Both fields attain their maximum values on the centerline and the soot number density peaks slightly earlier, at $x \approx 350 \mathrm{~mm}$, than the soot volume density $(x \approx 475 \mathrm{~mm})$. These observations indicate that nucleation is more vigorous at distances closer to the nozzle, yielding to soot surface growth further downstream. Furthermore, soot oxidation appears to act more effectively on the number density, while soot volume fraction shows a slightly delayed response. In view of the soot particle size distributions analyzed below (Figure 8), this may be the case because most soot particles persist in the nuclei size range and are, hence, rapidly removed by oxidation. By contrast, the larger soot particles which contribute significantly to the soot volume fraction can resist an oxidative gas composition for longer. Beyond the stoichiometric mixture fraction contour most soot has been oxidized and both the soot number density and volume fraction are close to vanishing.

In Figure 6, the centerline profiles of the predicted and experimentally measured mean soot volume 


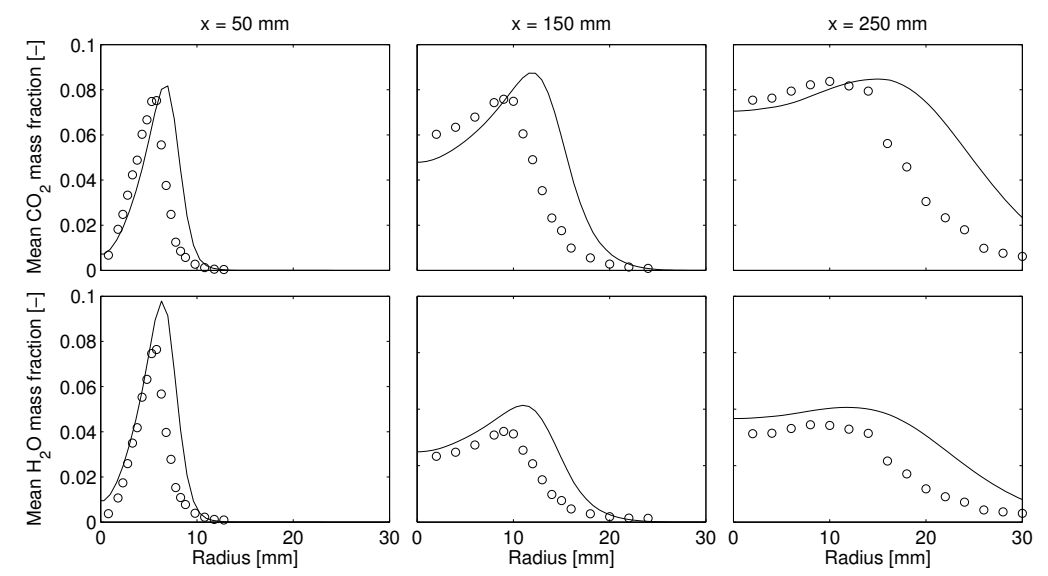

(a) $\mathrm{CO}_{2}$ and $\mathrm{H}_{2} \mathrm{O}$

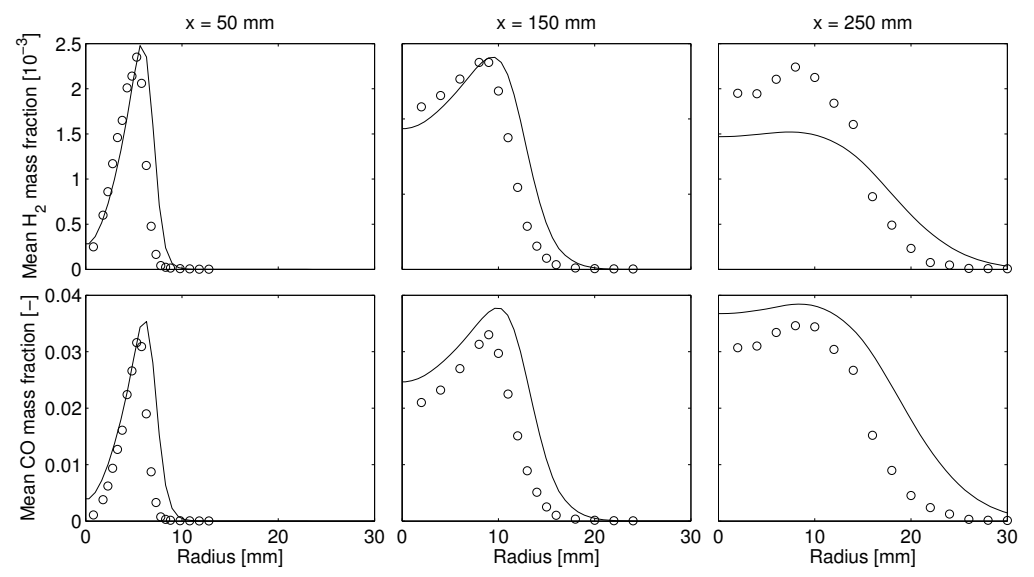

(b) $\mathrm{H}_{2}$ and $\mathrm{CO}$

Figure 4: Comparing radial profiles of selected mean species mass fractions (lines) with experimental measurements (symbols) at $50 \mathrm{~mm}, 150 \mathrm{~mm}$ and $250 \mathrm{~mm}$ above the nozzle exit plane.

fraction are compared. Here, the LES-PBE-PDF results indicate that soot formation commences much further upstream than was observed experimentally and, quantitatively, the mean soot volume fraction is underpredicted by one order of magnitude. A similar early onset and decay of soot formation was observed by Mueller and Pitsch [48] and Donde et al. [14], albeit for a soot model based on the hybrid method of moments and gas phase/soot kinetics which differed from the ones we employ here. These authors attributed the early onset to uncertainties in the soot formation kinetics.

In the context of a RANS-presumed $p d f$ approach based on the semi-empirical soot model of Brookes and Moss [10], Reddy et al. [64] found that the upstream shift of the centerline soot volume fraction profile can be remedied by computing the $\mathrm{OH}$ and $\mathrm{O}$ radical concentrations from equilibrium and partial equilibrium relations independent of the governing gas phase reaction mechanism and the turbulencechemistry interaction model. Since the semi-empirical model of Brookes and Moss [10] is based on $\mathrm{C}_{2} \mathrm{H}_{2}$, this is, possibly, related to the strong dependency of the $\mathrm{C}_{2} \mathrm{H}_{2}$ yield on the concentrations of $\mathrm{OH}$ and $\mathrm{O}$ which may be increased by the equilibrium and partial equilibrium chemistry in the nearfield. Reddy and De [63] and Reddy et al. [62, 64] further assessed the influence of different radiation models, but found that, while the peak soot volume fraction on the centerline can vary significantly, its location changes only slightly.

If we set aside the assumption of (partial) equilibrium $\mathrm{OH}$ and $\mathrm{O}$ concentrations, then both the present and previous modelling attempts of the Delft III flame predict an early onset and termination of soot formation. Since the modification employed by Reddy and De [63] and Reddy et al. [64] affects both the gas phase chemistry and overrides the turbulence-chemistry submodel, this indicates that either aspect may be held accountable. On the other hand, comparing our prediction and previous results $[14,48,64]$ 


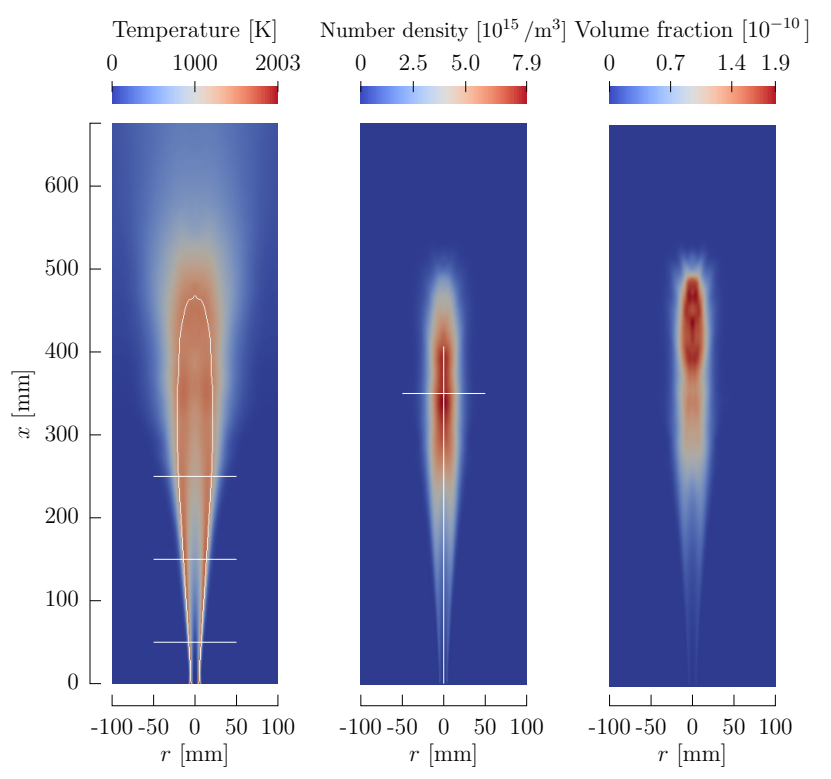

Figure 5: Contour plots of the mean temperature (left), soot number density (center) and soot volume fraction (right) computed from the LES-PBE-PDF model. The white contour in the left panel indicates stoichiometric mixture fraction values and the horizontal white lines show the near-field measurement stations at $50 \mathrm{~mm}, 150 \mathrm{~mm}$ and $250 \mathrm{~mm}$ above the nozzle. In the center panel, the white horizontal and vertical lines indicate the locations for which the instantaneous soot size distributions are shown in Figure 7.

for the soot volume fraction indicates that closures of the turbulence-soot formation interaction and the soot kinetics do not have as large an influence on the location of maximum soot volume fraction, albeit being important for quantitative differences. Consequently, it seems possible that there is a physical effect which is relevant for soot formation in methane-air combustion at moderate Reynolds numbers, but which both our attempt and previous investigations of soot formation in the Delft III flame omitted. Such an effect could be related to differential diffusion within the gas phase or differential micromixing between gas phase scalars and soot. For sooting ethylene flames at larger Reynolds numbers, Xuan and Blanquart [73] and Koo et al. [35] obtained very good predictions of soot volume fraction using LES-models similar to the one proposed by Mueller and Pitsch [48]. In this light the 'missing' physical effect, may turn less important under these conditions.

Apart from the upstream shift of the maximum soot volume fraction, our results in Figure 6 are characterized by an almost immediate onset of soot formation in the nearfield and a rather abrupt termination about $500 \mathrm{~mm}$ above the nozzle. The early onset of soot formation reflects the abundance of $\mathrm{C}_{2} \mathrm{H}_{2}$ and the large underprediction of soot volume fraction as well as the rapid decline in the vicinity of stoichiometric conditions indicates that soot oxidation is rather vigorous, potentially catalyzed by the temperature overprediction. Attili et al. [5] pointed out that soot surface growth takes place on much larger time scales than soot oxidation; in this light, it is possible that the residence time of soot pockets in flame regions which favour surface growth is too short, perhaps owing to limitations in the spatial LES resolution.

In order to elucidate the preferential location of soot relative to the flame front, we analyze the conditional soot number density and volume fraction in mixture fraction space for three different flame cross-sections in Figure 7. Here, the scatter represents instantaneous values sampled at several different time points, while the solid lines indicate time averages. The vertical dashed lines, furthermore, indicate the stoichiometric mixture fraction value of 0.0705. In terms of both instantaneous scatter and mean values the soot number density and volume fraction correlate well in mixture fraction space, reflecting the dominance of nucleation mentioned above. The instantaneous scatter covers a wide range of number density and volume fraction values on the rich side of the flame, emphasizing that soot number density and soot volume fraction may not be uniquely related to mixture fraction. On the lean side, by contrast, some scatter remains, but the number density and volume fraction values here are significantly reduced. 


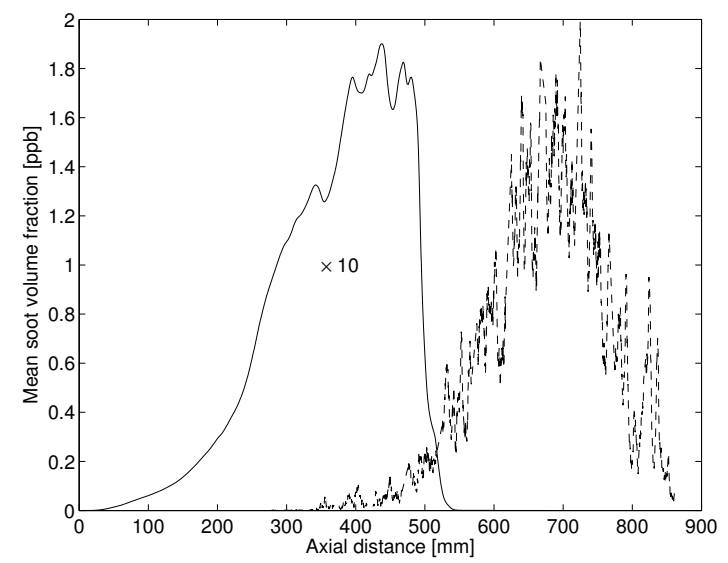

Figure 6: Comparing the mean soot volume fraction along the centerline (solid line) with the experimentally determined values (dashed line). Here, the LES-PBE-PDF predictions are scaled by a factor of 10.
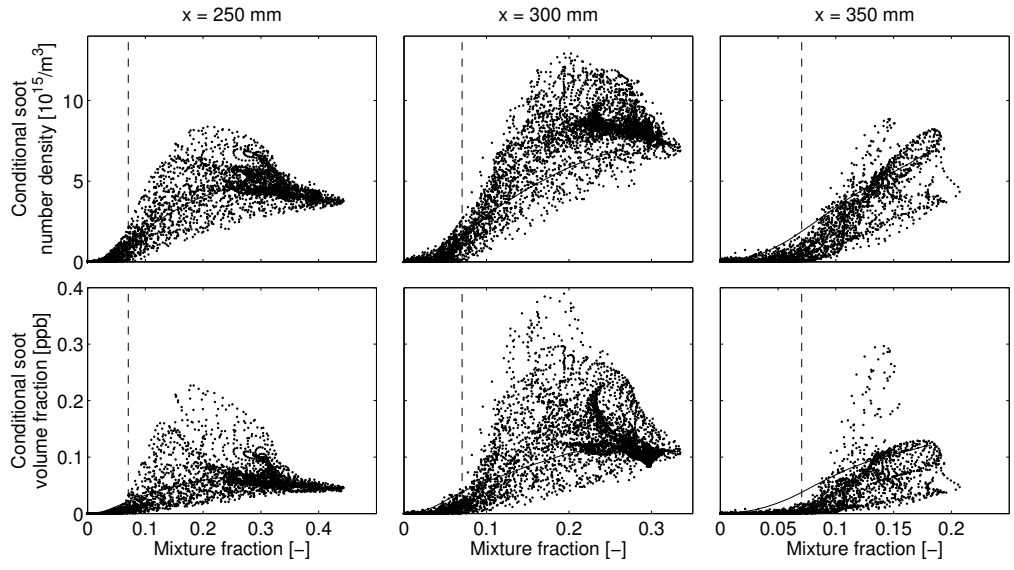

Figure 7: Instantaneous (scatter) and mean (solid lines) values of soot number density (top) and soot volume fraction (bottom) conditioned on mixture fraction at $250 \mathrm{~mm}, 300 \mathrm{~mm}$ and $350 \mathrm{~mm}$ above the nozzle. The vertical dashed lines indicate the stoichiometric mixture fraction of 0.0705 .

Possibly, oxidation is enhanced by the micromixing model whose rate applies, at the moment, equally to the gas phase scalars and to soot. Further downstream, both soot number density and volume fraction shift towards leaner mixture fraction values as the flame tip is approached. The soot that persists here at low mixture fraction values is completely oxidized away by $x=550 \mathrm{~mm}$.

Figure 8 depicts the instantaneous soot particle size distributions along the centerline of the flame and across the flame at the axial distance of maximum soot number density $(x=350 \mathrm{~mm})$. The grid lines which emanate from the particle size coordinate axis illustrate the grid adaptivity in particle size space. While the grid nodes are rather evenly spaced on a logarithmic scale at large particle sizes, the majority of nodes have been drawn into the vicinity of the mean nuclei size at $2.5 \mathrm{~nm}$ and maintain an accurate resolution of the sharp rise and decline in particle number density along the particle size coordinate. Both along and across the flame, the soot particle size distributions do not vary significantly in shape such that the grid nodes in particle size space display only very little spatial variability here. Throughout, the soot particle size distribution remains unimodal and, at the largest values of total soot number density and volume fraction, remains dominated by nucleation. In line with our observations for Figure 5 , the particle number density per unit of length in particle size space takes on maximum values near the centerline and rapidly decreases further outwards.

In Table 2, we compare the average runtimes of the fractional steps for the stochastic scalars and the 


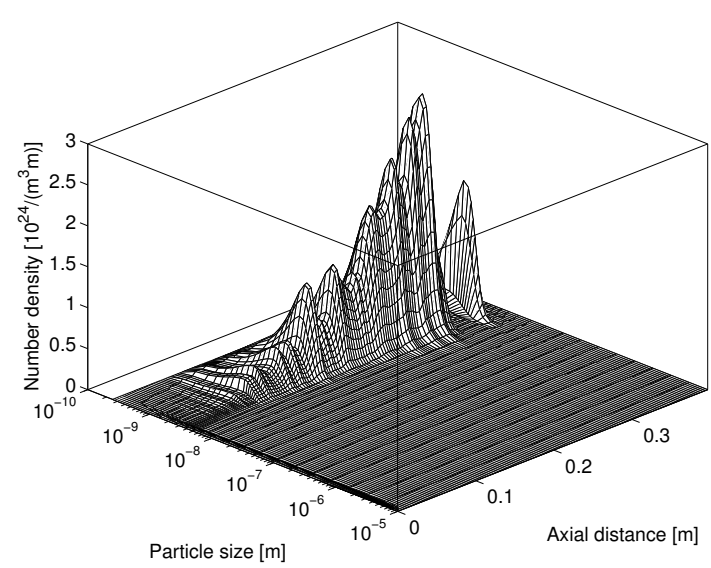

(a) Along the flame centerline

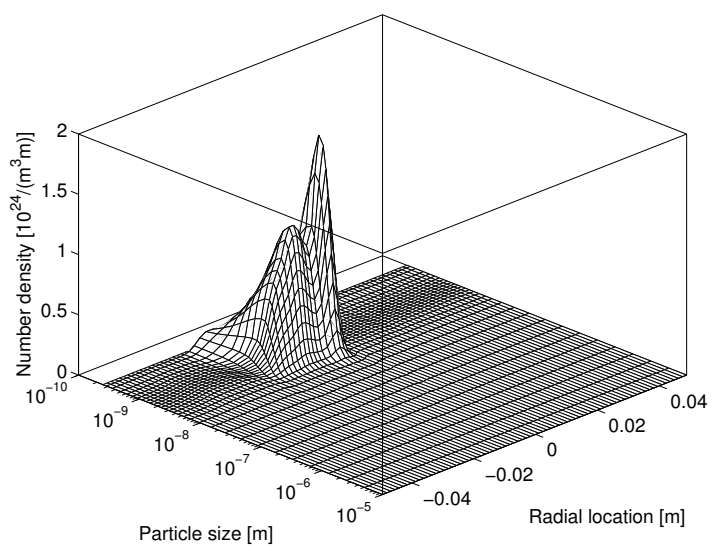

(b) Across the flame at $x=350 \mathrm{~mm}$

Figure 8: Instantaneous soot particle size distributions both along the flame centerline and across the flame at the axial distance of maximum mean soot number density. The spatial coordinate in the left panel runs along the vertical white line depicted in the central panel of Figure 5, while the spatial coordinate in the right panel corresponds to the horizontal white line.

\begin{tabular}{lc}
\hline \multicolumn{1}{c}{ Physical process } & Average runtime $[\mathrm{s}]$ \\
\hline Flow field & 1.94 \\
Scalar convection/diffusion & 6.89 \\
Mixing & 1.017 \\
Gas phase reaction & 10.85 \\
Particle phase reaction & 9.11 \\
\hline All processes & 33.54 \\
\hline
\end{tabular}

Table 2: Average runtimes for advancing the LES-PBE-PDF model by one time step $\left(\Delta t=1.2 \times 10^{-6} \mathrm{~s}\right)$ on 4 nodes (96 MPI processes) of a Cray XC30 Supercomputer (ARCHER UK).

flow solver on 4 nodes of a Cray XC30 Supercomputer (ARCHER UK). Here, the reaction fractional step for approximately $35.0 \%$ of reacting fluid cells takes slightly longer to execute than the PBE fractional step (including radiation, species consumption/release and particle size grid adaptation) which is called on all finite volume cells due to grid adaptivity. The PBE fractional step thus consumes about $27.2 \%$ of the overall runtime per time step, approximately matching the time fraction of $26.3 \%$ jointly required by scalar convection/diffusion and the flow solver.

The time measurements in Table 2 indicate that the combined LES-PBE-PDF approach is computationally feasible on modest resources of a modern computing system and that, compared to the gas phase reaction step, the PBE step does not significantly increase the overall runtime. Our observations thus demonstrate that a detailed resolution of particle size space within each fluid cell is viable and even computationally efficient. By contrast, the majority of studies investigating soot formation in turbulent flames have favoured moment based methods, at least in part, due to the concern that a detailed PBE model would incur excessive computational costs.

\section{Conclusions}

In the present article, we incorporated the PBE as a Eulerian description for the evolution of a polydispersed particulate phase into an LES model of a turbulent reacting flow with variable density. An important application of our approach is the prediction of soot particle size distributions in turbulent hydrocarbon flames. In order to resolve the turbulence-chemistry/particle formation interaction, we obtained an evolution equation for the filtered one-point, one-time joint $p d f$ of the instantaneous reactive scalars and particle number density. Here, turbulent transport was closed by a gradient diffusion hy- 
pothesis, while two-point correlations of the reactive scalars and number density were replaced by a micromixing model that accounts for differential diffusion between the gas and particle phases.

Numerically, the joint scalar-number density $p d f$ transport equation was solved using the method of Eulerian stochastic fields. In the context of LES, this approach has the advantage that its spatial resolution is independent of the large-scale flow structures - as opposed to stochastic particle based solvers, for instance. The stochastic process governing the stochastic fields is constructed such that, in a statistical sense, the evolution of the joint scalar-number density $p d f$ is reproduced.

An important feature of our numerical solution scheme is the adaptive grid discretization of particle size space. This technique allows particle size distributions which vary largely in shape and width across the flow domain, potentially including sharp peaks or near-discontinuities, to be represented with similar accuracy, while maintaining consistency of the particle size discretization across the flow domain. Additionally, the number of grid points in particle size space is significantly reduced as compared to fixed grid discretization approaches [68].

The combined LES-PBE-PDF model was applied to model soot formation in the Delft III diffusion flame. Here, kinetic rate expressions for soot nucleation and growth were adopted from previous laminar diffusion flame calculations and both species depletion as well as radiation based on the hypothesis of optical thinness were included. At present, the formation of chain-like soot aggregates is not considered such that our soot phase is described solely in terms of spherical primary particles. Similarly, the coagulation of nascent soot particles is omitted, although we intend to incorporate coagulation in future times. The gas phase kinetics were represented by the detailed GRI 1.2 reaction mechanism.

In the nearfield of the Delft III flame both the mean velocity and gas phase scalars agree reasonably well with measurements. The overprediction on the lean side here seems to be due to limitations of the spatial resolution which may render the turbulence closures less accurate. Similar to previous LES investigations of the Delft III flame, the soot volume fraction peaks further upstream than in the experimental observations. Additionally, soot volume fractions are notably underpredicted. However, except for the upstream shift and a slightly early onset of soot formation, the centerline soot volume fraction profile compares well qualitatively with the experimental data. Predictions of the soot particle size distribution reflect the dominance of soot nucleation and oxidation and their influence on the shape of the local particle size distribution. In terms of performance, we found the solver for the PBE fractional step to only consume a modest fraction of the average runtime per time step.

Since most of the computational cost remains concentrated in the gas phase reaction step, we aim at incorporating an apt tabulation technique in the near future [17]. Additionally, the more recently proposed soot kinetics are based on PAH chemistry and, hence, require a more comprehensive gas phase reaction mechanism. By applying a mechanism reduction technique, see, for example, Reference [34], the number of gas phase scalars may be manageably reduced. On part of the soot formation processes, the present LES-PBE-PDF framework is also suited for accommodating coagulation of incipient soot particles. As a final model enhancement we mention the inclusion of gas phase differential diffusion as well as the prescription of a different micromixing frequency for each gas phase scalar and for soot.

In conclusion, our investigation has demonstrated that modelling soot formation in a turbulent flame based on the LES-PBE-PDF approach is not only advantageous seeing as any soot kinetics can be accommodated without approximation and the entire particle size distribution is predicted, but also computationally efficient.

\section{Acknowledgements}

Fabian Sewerin gratefully acknowledges the support from the Imperial College PhD Scholarship Scheme. This work used the ARCHER UK National Supercomputing Service (http://www.archer.ac.uk).

\section{Appendix A An evolution equation for the transition $p d f$ asso- ciated with the stochastic fields}

In this section, an evolution equation is obtained for the transition $p d f h(\mathbf{z}, t ; \mathbf{x})$ associated with the stochastic process $\boldsymbol{\theta}(t ; \cdot, \mathbf{x})$ that evolves according to Eq. (37) subject to the initial condition $\boldsymbol{\theta}\left(t_{0} ; \cdot, \mathbf{x}\right)=$ $\left(\mathbf{Y}_{0}(\mathbf{x}), N_{\rho, 0}(\cdot, \mathbf{x})\right)$. Following the rationale outlined by Sewerin and Rigopoulos $[69$, Appendix B], we 
introduce the fine-grained density function $h^{\prime}(\mathbf{z}, t ; \mathbf{x})$ associated with $\boldsymbol{\theta}(t ; \cdot, \mathbf{x})$,

$$
h^{\prime}(\mathbf{z}, t ; \mathbf{x})=\delta(\mathbf{z}-\boldsymbol{\theta}(t ; \cdot, \mathbf{x})),
$$

and recall that applying the expectation operator $\langle\cdot\rangle$ to Eq. (47) yields the transition $p d f h(\mathbf{z}, t ; \mathbf{x})$,

$$
h(\mathbf{z}, t ; \mathbf{x})=\left\langle h^{\prime}(\mathbf{z}, t ; \mathbf{x})\right\rangle=\langle\delta(\mathbf{z}-\boldsymbol{\theta}(t ; \cdot, \mathbf{x}))\rangle .
$$

The spatial derivatives of $h^{\prime}(\mathbf{z}, t ; \mathbf{x})$ are given by

$$
\frac{\partial h^{\prime}}{\partial x_{j}}=-\sum_{i=1}^{n_{s}+1} \frac{\partial h^{\prime}}{\partial z_{i}} \frac{\partial \theta_{i}}{\partial x_{j}}, \quad j=1, \ldots, 3
$$

Since the fine-grained density depends on time through the stochastic process $\boldsymbol{\theta}(t ; \cdot, \mathbf{x})$ (Eq. (47)), the temporal derivative of $h^{\prime}(\mathbf{z}, t ; \mathbf{x})$ can be obtained from Itô's formula [56, Section 3.4],

$$
\frac{\partial h^{\prime}}{\partial t}=-\sum_{i=1}^{n_{s}+1} \frac{\partial h^{\prime}}{\partial z_{i}} \frac{\partial \theta_{i}}{\partial t}+\frac{1}{2} \sum_{i, j=1}^{n_{s}+1} \sum_{k=1}^{3} \frac{\partial^{2} h^{\prime}}{\partial z_{i} \partial z_{j}} \sigma_{i k} \sigma_{j k}
$$

where $\sigma_{i k}\left(\partial \theta_{i} / \partial x_{k}, \mathbf{x}, t\right)$ represents the diffusion coefficient associated with Eq. (37),

$$
\sigma_{i k}\left(\frac{\partial \theta_{i}}{\partial x_{k}}, \mathbf{x}, t\right)=-\sqrt{2 \Gamma(\mathbf{x}, t)} \frac{\partial \theta_{i}}{\partial x_{k}} .
$$

Introducing Eqs. (37) and (51) into Eq. (50) and taking into account Eq. (49) as well as the identity

$$
\sum_{i=1}^{n_{s}+1} \sum_{k=1}^{3}\left\{\bar{\rho} \Gamma \frac{\partial \theta_{i}}{\partial x_{k}} \sum_{j=1}^{n_{s}+1} \frac{\partial^{2} h^{\prime}}{\partial z_{j} \partial z_{i}} \frac{\partial \theta_{j}}{\partial x_{k}}-\frac{\partial h^{\prime}}{\partial z_{i}} \frac{\partial}{\partial x_{k}}\left(\bar{\rho} \Gamma \frac{\partial \theta_{i}}{\partial x_{k}}\right)\right\}=\sum_{k=1}^{3} \frac{\partial}{\partial x_{k}}\left(\bar{\rho} \Gamma \frac{\partial h^{\prime}}{\partial x_{k}}\right)
$$

leads to the following evolution equation for the fine-grained $p d f h^{\prime}(\mathbf{z}, t ; \mathbf{x})$

$$
\begin{aligned}
\bar{\rho} \frac{\partial h^{\prime}}{\partial t}+\sum_{j=1}^{3} \bar{\rho} \tilde{u}_{j} \frac{\partial h^{\prime}}{\partial x_{j}} & =\sum_{k=1}^{3} \frac{\partial}{\partial x_{k}}\left(\bar{\rho} \Gamma \frac{\partial h^{\prime}}{\partial x_{k}}\right)-\bar{\rho} \sqrt{2 \Gamma} \sum_{k=1}^{3} \frac{\partial h^{\prime}}{\partial x_{k}} \dot{W}_{k}(t) \\
& -\sum_{i=1}^{n_{s}+1} \frac{\partial h^{\prime}}{\partial z_{i}} \bar{\rho}\left(s_{i}(\cdot, \boldsymbol{\theta})+m_{i}(\mathbf{x}, t, \boldsymbol{\theta})\right) .
\end{aligned}
$$

By applying the expectation operator $\langle\cdot\rangle$ to Eq. (53), we obtain on account of the commutation property of $\langle\cdot\rangle$, the $\langle\cdot\rangle$-analogue of Eq. (25) and by the martingale property of the Itô stochastic integral

$$
\bar{\rho} \frac{\partial h}{\partial t}+\sum_{j=1}^{3} \bar{\rho} \tilde{u}_{j} \frac{\partial h}{\partial x_{j}}=\sum_{k=1}^{3} \frac{\partial}{\partial x_{k}}\left(\bar{\rho} \Gamma \frac{\partial h}{\partial x_{k}}\right)-\sum_{i=1}^{n_{s}+1} \frac{\partial}{\partial z_{i}}\left(\bar{\rho} h\left(s_{i}(\cdot, \mathbf{z})+m_{i}(\mathbf{x}, t, \mathbf{z})\right)\right) .
$$

This evolution equation corresponds to our physical modelled $p d f$ transport equation for $\tilde{f}(\mathbf{z} ; \mathbf{x}, t)$ given in Eqs. (28), (31) and (33).

\section{References}

[1] (2003). http://www.sandia.gov/tnf/radiation.html.

[2] Akridis, P. (2016). Coupled CFD-Population Balance Modelling of Soot Formation in Laminar and Turbulent Flames. PhD thesis, Imperial College London.

[3] Akridis, P. and Rigopoulos, S. (2015). Modelling of soot formation in a turbulent diffusion flame using a comprehensive CFD-PBE model with full chemistry. In Proceedings of the European Combustion Meeting 2015, Budapest, Hungary. 
[4] Akridis, P. and Rigopoulos, S. (2017). Modelling of soot formation in laminar diffusion flames using a comprehensive CFD-PBE model with detailed gas-phase chemistry. Combustion Theory and Modelling, $21(1): 35-48$.

[5] Attili, A., Bisetti, F., Mueller, M. E., and Pitsch, H. (2014). Formation, growth, and transport of soot in a three-dimensional turbulent non-premixed jet flame. Combustion and Flame, 161(7):1849-1865.

[6] Ayache, S. and Mastorakos, E. (2012). Conditional moment closure/large eddy simulation of the Delft-III natural gas non-premixed jet flame. Flow, Turbulence and Combustion, 88(1-2):207-231.

[7] Bai, X. S., Balthasar, M., Mauss, F., and Fuchs, L. (1998). Detailed soot modeling in turbulent jet diffusion flames. Symposium (International) on Combustion, 27(1):1623-1630.

[8] Bisetti, F., Attili, A., and Pitsch, H. (2014). Advancing predictive models for particulate formation in turbulent flames via massively parallel direct numerical simulations. Philosophical Transactions of the Royal Society A: Mathematical, Physical and Engineering Sciences, 372(2022).

[9] Bressloff, N. W., Moss, J. B., and Rubini, P. A. (1996). CFD prediction of coupled radiation heat transfer and soot production in turbulent flames. Symposium (International) on Combustion, $26(2): 2379-2386$.

[10] Brookes, S. J. and Moss, J. B. (1999). Predictions of soot and thermal radiation properties in confined turbulent jet diffusion flames. Combustion and Flame, 116(4):486-503.

[11] Di Veroli, G. Y. and Rigopoulos, S. (2010). Modeling of turbulent precipitation: A transported population balance-PDF method. AIChE Journal, 56(4):878-892.

[12] Di Veroli, G. Y. and Rigopoulos, S. (2011). Modeling of aerosol formation in a turbulent jet with the transported population balance equation-probability density function approach. Physics of Fluids, 23(4):043305.

[13] Dodoulas, I. A. and Navarro-Martinez, S. (2015). Analysis of extinction in a non-premixed turbulent flame using large eddy simulation and the chemical explosion mode analysis. Combustion Theory and Modelling, 19(1):107-129.

[14] Donde, P., Raman, V., Mueller, M. E., and Pitsch, H. (2013). LES/PDF based modeling of sootturbulence interactions in turbulent flames. Proceedings of the Combustion Institute, 34(1):1183-1192.

[15] Fox, R. O. (1998). On the relationship between Lagrangian micromixing models and computational fluid dynamics. Chemical Engineering and Processing: Process Intensification, 37(6):521-535.

[16] Fox, R. O. (2003). Computational Models for Turbulent Reacting Flows. Cambridge University Press.

[17] Franke, L. L. C., Chatzopoulos, A. K., and Rigopoulos, S. (2017). Tabulation of combustion chemistry via artificial neural networks (ANNs): Methodology and application to LES-PDF simulation of Sydney flame L. Combustion and Flame, 185:245-260.

[18] Frenklach, M., Wang, H., Goldenberg, M., Smith, G. P., Golden, D., Bowman, C. T., Hanson, R. K., Gardiner, W. C., and Lissianski, V. (1995a). GRI-Mech-An Optimized Detailed Chemical Reaction Mechanism for Methane Combustion. Topical Report No. GRI-95/0058, Gas Research Institute.

[19] Frenklach, M., Wang, H., Yu, C.-L., Goldenberg, M., Bowman, C. T., Hanson, R. K., Davidson, D. F., Chang, E. J., Smith, G. P., Golden, D. M., Gardiner, W. C., and Lissianski, V. (1995b). GRI-Mech 1.2.

[20] Gore, J. P., Ip, U.-S., and Sivathanu, Y. R. (1992). Coupled structure and radiation analysis of acetylene/air flames. Journal of Heat Transfer, 114:487-493.

[21] Grosschmidt, D., Habisreuther, P., and Bockhorn, H. (2007). Calculation of the size distribution function of soot particles in turbulent diffusion flames. Proceedings of the Combustion Institute, 31(1):657665 .

[22] Grosshandler, W. L. (1993). RADCAL: A narrow-band model for radiation calculations in a combustion environment. Nist technical note 1402, National Institute of Standards and Technology. 
[23] Hairer, E., Nørsett, S. P., and Wanner, G. (1993). Solving Ordinary Differential Equations I: Nonstiff Problems, volume 8 of Springer Series in Computational Mathematics. Springer-Verlag, Berlin, Heidelberg.

[24] Hall, R. J., Smooke, M. D., and Colket, M. B. (1997). Predictions of soot dynamics in opposed jet diffusion flames. In Sawyer, R. F. and Dryer, F. L., editors, Physical and Chemical Aspects of Combustion: A Tribute to Irvin Glassman, pages 189-230. Combustion Science and Technology Book Series, Gordon \& Breach, Langhorne, PA.

[25] Harris, S. J. and Weiner, A. M. (1983). Surface growth of soot particles in premixed ethylene/air flames. Combustion Science and Technology, 31(3-4):155-167.

[26] Hauke, G. and Valiño, L. (2004). Computing reactive flows with a field Monte Carlo formulation and multi-scale methods. Computer Methods in Applied Mechanics and Engineering, 193(15-16):1455-1470.

[27] Hulburt, H. M. and Katz, S. (1964). Some problems in particle technology: A statistical mechanical formulation. Chemical Engineering Science, 19(8):555-574.

[28] Hunt, A., Abraham, J. L., Judson, B., and Berry, C. L. (2003). Toxicologic and epidemiologic clues from the characterization of the 1952 London smog fine particulate matter in archival autopsy lung tissues. Environmental Health Perspectives, 111(9):1209-1214.

[29] Jones, W. P. (2002). The joint scalar probability density function method. In Launder, B. E. and Sandham, N., editors, Closure Strategies for Turbulent and Transitional Flows, chapter 20, pages 582-625. Cambridge University Press.

[30] Jones, W. P. and Navarro-Martinez, S. (2007). Large eddy simulation of autoignition with a subgrid probability density function method. Combustion and Flame, 150(3):170-187.

[31] Jones, W. P., Navarro-Martinez, S., and Röhl, O. (2007). Large eddy simulation of hydrogen auto-ignition with a probability density function method. Proceedings of the Combustion Institute, 31(2):1765-1771.

[32] Jones, W. P. and Prasad, V. N. (2010). Large eddy simulation of the Sandia flame series (D-F) using the Eulerian stochastic field method. Combustion and Flame, 157(9):1621-1636.

[33] Jones, W. P. and Prasad, V. N. (2011). LES-PDF simulation of a spark ignited turbulent methane jet. Proceedings of the Combustion Institute, 33(1):1355-1363.

[34] Koniavitis, P., Rigopoulos, S., and Jones, W. P. (2017). A methodology for derivation of RCCEreduced mechanisms via CSP. Combustion and Flame, 183:126-143.

[35] Koo, H., Hassanaly, M., Raman, V., Mueller, M. E., and Geigle, K. P. (2016). Large-eddy simulation of soot formation in a model gas turbine combustor. Journal of Engineering for Gas Turbines and Power, 139(3):031503.

[36] Koren, B. (1993). A robust upwind discretization method for advection, diffusion and source terms. In Vreugdenhil, C. B. and Koren, B., editors, Numerical Methods for Advection-Diffusion Problems, volume 45 of Notes on Numerical Fluid Mechanics and Multidisciplinary Design, pages 117-138. Vieweg Verlag.

[37] Kronenburg, A., Bilger, R., and Kent, J. H. (2000). Modeling soot formation in turbulent methaneair jet diffusion flames. Combustion and Flame, 121(1-2):24-40.

[38] Lilly, D. K. (1967). The representation of small scale turbulence in numerical simulation experiments. Technical Report 281, National Center for Atmospheric Research (NCAR).

[39] Lindstedt, R. P. and Louloudi, S. A. (2005). Joint-scalar transported PDF modeling of soot formation and oxidation. Proceedings of the Combustion Institute, 30(1):775-783.

[40] Liu, F., Guo, H., Smallwood, G., and Gülder, Ö. (2003). Numerical modelling of soot formation and oxidation in laminar coflow non-smoking and smoking ethylene diffusion flames. Combustion Theory and Modelling, 7(2):301-315. 
[41] Marchisio, D. L. and Fox, R. O. (2013). Computational Models for Polydisperse Particulate and Multiphase Systems. Cambridge Series in Chemical Engineering. Cambridge University Press, Cambridge, New York.

[42] McDermott, R. and Pope, S. B. (2007). A particle formulation for treating differential diffusion in filtered density function methods. Journal of Computational Physics, 226(1):947-993.

[43] Merci, B., Naud, B., and Roekaerts, D. (2005). Flow and mixing fields for transported scalar PDF simulations of a piloted jet diffusion flame ('Delft flame III'). Flow, Turbulence and Combustion, 74(3):239-272.

[44] Merci, B., Roekaerts, D., and Naud, B. (2006). Study of the performance of three micromixing models in transported scalar PDF simulations of a piloted jet diffusion flame ("Delft flame III"). Combustion and Flame, 144(3):476-493.

[45] Metternich, M., Kollmann, W., Kennedy, I. M., and Chen, J.-Y. (1991). PDF prediction of sooting turbulent flames. In 29th Aerospace Sciences Meeting, Reno, NV, USA.

[46] Moss, J. B., Stewart, C. D., and Syed, K. J. (1989). Flowfield modelling of soot formation at elevated pressure. Symposium (International) on Combustion, 22(1):413-423.

[47] Mueller, M. E., Blanquart, G., and Pitsch, H. (2009). Hybrid method of moments for modeling soot formation and growth. Combustion and Flame, 156(6):1143-1155.

[48] Mueller, M. E. and Pitsch, H. (2012). LES model for sooting turbulent nonpremixed flames. Combustion and Flame, 159(6):2166-2180.

[49] Mueller, M. E. and Raman, V. (2014). Effects of turbulent combustion modeling errors on soot evolution in a turbulent nonpremixed jet flame. Combustion and Flame, 161(7):1842-1848.

[50] Mustata, R., Valiño, L., Jiménez, C., Jones, W. P., and Bondi, S. (2006). A probability density function Eulerian Monte Carlo field method for large eddy simulations: Application to a turbulent piloted methane/air diffusion flame (Sandia D). Combustion and Flame, 145(1-2):88-104.

[51] Nagle, J. and Strickland-Constable, R. F. (1962). Oxidation of carbon between 1000-2000 C. In Proceedings of the fifth Carbon Conference, volume 1, pages 154-164, New York. Pergamon.

[52] Navarro-Martinez, S. and Rigopoulos, S. (2012). Differential diffusion modelling in LES with RCCEreduced chemistry. Flow, Turbulence and Combustion, 89(2):311-328.

[53] Netzell, K., Lehtiniemi, H., and Mauss, F. (2007). Calculating the soot particle size distribution function in turbulent diffusion flames using a sectional method. Proceedings of the Combustion Institute, 31(1):667-674.

[54] Neuber, G., Kronenburg, A., Stein, O. T., and Cleary, M. J. (2017). MMC-LES modelling of droplet nucleation and growth in turbulent jets. Chemical Engineering Science, 167:204-218.

[55] Nooren, P. A., Versluis, M., van der Meer, T. H., Barlow, R. S., and Frank, J. H. (2000). RamanRayleigh-LIF measurements of temperature and species concentrations in the Delft piloted turbulent jet diffusion flame. Applied Physics B, 71(1):95-111.

[56] Pavliotis, G. A. (2014). Stochastic Processes and Applications. Springer, New York.

[57] Peeters, T. W. J., Stroomer, P. P. J., de Vries, J. E., Roekaerts, D. J. E. M., and Hoogendoorn, C. J. (1994). Comparative experimental and numerical investigation of a piloted turbulent natural-gas diffusion flame. Symposium (International) on Combustion, 25(1):1241-1248.

[58] Pope, S. B. (1985). PDF methods for turbulent reactive flows. Progress in Energy and Combustion Science, 11(2):119-192.

[59] Pope, S. B. (1991). Computations of turbulent combustion: Progress and challenges. Symposium (International) on Combustion, 23(1):591-612. 
[60] Qamar, N. H., Alwahabi, Z. T., Chan, Q. N., Nathan, G. J., Roekaerts, D., and King, K. D. (2009). Soot volume fraction in a piloted turbulent jet non-premixed flame of natural gas. Combustion and Flame, 156(7):1339-1347.

[61] Raman, V. and Fox, R. O. (2016). Modeling of fine-particle formation in turbulent flames. Annual Review of Fluid Mechanics, 48:159-190.

[62] Reddy, B. M., De, A., and Yadav, R. (2016). Numerical investigation of soot formation in turbulent diffusion flame with strong turbulence-chemistry interaction. Journal of Thermal Science and Engineering Applications, 8(1):011001.

[63] Reddy, M. and De, A. (2014). Numerical investigation of soot formation in turbulent diffusion flames using Moss-Brookes model. In ASME 2014 Gas Turbine India Conference, GTINDIA 2014, New Delhi, India.

[64] Reddy, M., De, A., and Yadav, R. (2015). Effect of precursors and radiation on soot formation in turbulent diffusion flame. Fuel, 148:58-72.

[65] Rigopoulos, S. (2007). PDF method for population balance in turbulent reactive flow. Chemical Engineering Science, 62(23):6865-6878.

[66] Rigopoulos, S. (2010). Population balance modelling of polydispersed particles in reactive flows. Progress in Energy and Combustion Science, 36(4):412-443.

[67] Sabel'nikov, V. and Soulard, O. (2005). Rapidly decorrelating velocity-field model as a tool for solving one-point Fokker-Planck equations for probability density functions of turbulent reactive scalars. Physical Review E, 72(1):016301.

[68] Sewerin, F. and Rigopoulos, S. (2017a). An explicit adaptive grid approach for the numerical solution of the population balance equation. Chemical Engineering Science, 168:250-270.

[69] Sewerin, F. and Rigopoulos, S. (2017b). An LES-PBE-PDF approach for modeling particle formation in turbulent reacting flows. Physics of Fluids, 29(10):105105.

[70] Smooke, M. D., Long, M. B., Connelly, B. C., Colket, M. B., and Hall, R. J. (2005). Soot formation in laminar diffusion flames. Combustion and Flame, 143(4):613-628.

[71] Smooke, M. D., McEnally, C. S., Pfefferle, L. D., Hall, R. J., and Colket, M. B. (1999). Computational and experimental study of soot formation in a coflow, laminar diffusion flame. Combustion and Flame, 117(1-2):117-139.

[72] Valiño, L. (1998). A field Monte Carlo formulation for calculating the probability density function of a single scalar in a turbulent flow. Flow, Turbulence and Combustion, 60(2):157-172.

[73] Xuan, Y. and Blanquart, G. (2015). Effects of aromatic chemistry-turbulence interactions on soot formation in a turbulent non-premixed flame. Proceedings of the Combustion Institute, 35(2):19111919.

[74] Young, K. J. and Moss, J. B. (1995). Modelling sooting turbulent jet flames using an extended flamelet technique. Combustion Science and Technology, 105(1-3):33-53.

[75] Zucca, A., Marchisio, D. L., Barresi, A. A., and Fox, R. O. (2006). Implementation of the population balance equation in CFD codes for modelling soot formation in turbulent flames. Chemical Engineering Science, 61(1):87-95. 